\title{
Boosting Memory Access Locality of the Spectral Element Method with Hilbert Space-Filling Curves
}

\author{
Roger R. F. Araújo ${ }^{a}$, Lutz Gross ${ }^{b}$ and Samuel Xavier-de-Souza ${ }^{c}$ \\ a Programa de Pós-Graduação em Engenharia Elétrica e de Computação, \\ Universidade Federal do Rio Grande do Norte, \\ Natal/RN, Brazil (e-mail: roger_rf@dca.ufrn.br) \\ b School of Earth and Environmental Sciences, \\ The University of Queensland, \\ Brisbane, Queensland, Australia (e-mail: l.gross@uq.edu.au) \\ c Departamento de Engenharia de Computação e Automação, \\ Universidade Federal do Rio Grande do Norte, \\ Natal/RN, Brazil (e-mail: samuel@dca.ufrn.br) \\ Corresponding author: \\ Roger R. F. Araújo (e-mail: roger_rf@dca.ufrn.br)
}

April 2021

\begin{abstract}
We propose an algorithm based on Hilbert spacefilling curves to reorder mesh elements in memory for use with the Spectral Element Method, aiming to attain fewer cache misses, better locality of data reference and faster execution. We present a technique to numerically simulate acoustic wave propagation in 2D domains using the Spectral Element Method, and discuss computational performance aspects of this procedure. We reorder mesh-related data via Hilbert curves to achieve sizable reductions in execution time under several mesh configurations in shared-memory systems. Our experiments show that the Hilbert curve approach works well with meshes of several granularities and also with small and large variations in element
\end{abstract}

sizes, achieving reductions between $9 \%$ and $25 \%$ in execution time when compared to three other ordering schemes.

Keywords - Hilbert space-filling curves, spectral element method, unstructured meshes, acoustic waves, wave propagation, parallel processing.

\section{Introduction}

The efficient solution of the wave equation in terms of accuracy and computational performance is a topic of recurrent interest. The Spectral Element Method (SEM) (Patera, 1984; Kopriva, 2009), a variant of the Finite Element Method (FEM) (Chaskalovic, 2008), has been successfully applied to various wave-related problems (Zampieri and Pavarino, 2006; Bakir and 
Nowack, 2012; Afanasiev et al., 2018). SEM has proven to be versatile when it comes to the equations it can solve and to the geometry of the underlying domain, including complex media interfaces (Komatitsch and Vilotte, 1998).

Both SEM and FEM discretize the domain as a mesh of interconnected elements to convert the target equation into a linear system in matrix form. FEM gives rise to sparse matrices, and the corresponding linear systems require specialized techniques to solve. In addition, the computational efforts to process those linear systems grow along with mesh resolution, posing a potential performance bottleneck. SEM avoids these complications by creating mass matrices that are diagonal by construction (Komatitsch and Tromp, 1999) when using explicit time integration schemes. Diagonal mass matrices are desirable, as they minimize numerical diffusion and avoid matrix lumping. Moreover, the resulting linear systems are straightforward to solve and well-suited to parallel processing.

SEM-based software implementations need to traverse mesh elements and their nodes as they march an equation through time. Irregular and non-local memory accesses can severely harm this process. The layout of data structures, the sequence of memory accesses and cache hierarchy utilization all influence the efficiency of those traversals and overall execution performance (Sastry et al., 2014). Improving spatial and temporal locality (Stallings, 2009) increases performance by avoiding cache misses.

In this work, we propose an approach based on Hilbert space-filling curves (SFCs) (Hilbert, 1891) to reorder mesh-related SEM data, mitigating irregular memory access and maximizing locality. We extended esys-escript (Schaa et al., 2016), an open-source mathematical modeling tool, to support this approach and developed an SEM-based 2D acoustic wave simulator. We conducted experiments to compare the proposed Hilbert curve approach to different strategies using homogeneous and heterogeneous meshes and found that it works noticeably better, showing solid performance improvements in meshes of different granularities and large variations in element sizes. Finally, we show that our SEM formulation reliably simulates wave propagation in a complex, multilayered geological structure.
In the next section, we review prior work on SEMbased Partial Differential Equation (PDE) solvers and the use of SFCs to improve memory efficiency. Section 3 describes how to generate standard Hilbert curves over squares, and a generalized approach that provides greater geometric flexibility. In Section 4 we present our formulation to solve the wave equation using SEM with unstructured meshes of triangular elements and an explicit time integration scheme. In the same Section, we propose the algorithm to reorder mesh-related data using generalized Hilbert curves for memory-efficient traversals. Section 5 discusses our implementation and optimization of the time integration scheme. In Section 6, we conduct performance benchmarks of our proposed implementation with several memory reordering strategies and present a realistic wave propagation example. In the final section, we draw some conclusions.

\section{Related Work}

Wave-type PDEs are essential in various fields such as acoustics, fluid dynamics and geophysics. Over time this resulted in the development of many wave equation solvers, of which we mention some SEM-based examples. Komatitsch and Vilotte (1998) solve elastic waves over unstructured meshes of quadrilaterals in 2D and hexahedra in 3D. Komatitsch and Tromp (1999) discuss the simulation of seismic waves in 3D with unstructured meshes of hexahedral elements. Komatitsch et al. (2000) use unstructured meshes of quadrilaterals in $2 \mathrm{D}$ and hexahedra in 3D to model wave propagation near a fluid-solid interface, taking into account the specific behavior of each medium. Mercerat et al. (2006) simulate elastic waves using unstructured meshes of triangles in $2 \mathrm{D}$, in a work that employs SEM but does not use diagonal mass matrices. The SPECFEM3D Cartesian software package (Komatitsch et al., 2012) simulates several wave types over unstructured meshes of hexahedra. The p 4est software library (Burstedde et al., 2011), while not an equation solver per se, offers optimized data structures to work with unstructured meshes and uses forests of quadtrees in 2D and octrees in 3D for local refinement of elements. Our proposed implementa- 
tion uses SEM to simulate acoustic wave propagation in $2 \mathrm{D}$ over unstructured meshes made of triangles.

Moving on to SFCs, they have the useful property of preserving spatial proximity between successive steps of the curve when marching through rectangles and hexahedra. This led to various applications in computer science that reorder memory or operations in the manner of SFC traversals to enhance cache utilization and memory efficiency. Voorhies uses Hilbert curves to reduce the number of paging operations required by computer screen scans when loading and discarding data related to objects randomly scattered across a screen (Arvo, 1991). Mellor-Crummey et al. (2001) employ SFCs to rearrange data and computations in particle problems, reducing cache misses and execution time. Sastry et al. (2014) perform mesh warping through Laplace's equations, and use Hilbert curves to reorder mesh elements and vertices to achieve fewer cache misses and faster execution speed. The aforementioned p4est library, whose main feature is parallel adaptive mesh refinement, is based on SFCs. We leverage a relatively recent formulation of generalized Hilbert curves to develop our algorithm to reorder mesh-related data.

\section{Boosting Locality with Space- Filling Curves}

According to the principle of locality of reference, when executing a program, a processor typically accesses the same memory areas repeatedly during short periods (Stallings, 2009). A proper distribution of data among and within processors increases the probability that, after blocks from main memory get written to cache, memory references that the program makes in the near future are already available in the cache lines, helping to improve performance.

Upon first loading a mesh into memory from an external source such as a data file, we cannot expect its elements and nodes to follow any particular order. As a solver program marches a wave equation through time using SEM, it needs to traverse all mesh elements, and all local nodes of each element, at each step of its time integration scheme. If entities whose coordinates are close together in space lie far apart in memory, traversing them in the time integration scheme results in poor data locality. We can better exploit locality of reference by reordering data or computations to follow the traversal path of SFCs, thus leveraging their proximity-preserving properties. Potential improvements are better cache usage, memory efficiency and execution time.

There are several SFC formulations we can use when developing memory reordering strategies, such as Peano curves (Peano, 1890), Z-order curves (Morton, 1966) and Hilbert curves (Hilbert, 1891). We focus our attention on Hilbert curves. Listing 1 shows a simple, recursive Python generator of standard Hilbert curves, adapted from Warren (2013). The curve runs through all integer $\left(x_{0}, x_{1}\right)$-coordinates inside the square of edge length $2^{a}-1$, where $a$ is the order of the curve. The hilbert() function continuously splits the square into smaller squares. The step() function takes a step toward each smaller square, updating variables $x_{0}, x_{1}$ and distance. $x_{0}$ and $x_{1}$ are the intermediate coordinates along the traversal, and distance is the accumulated distance. Figure 1 illustrates the traversal, displaying similar colors in the vicinity of intermediate steps.

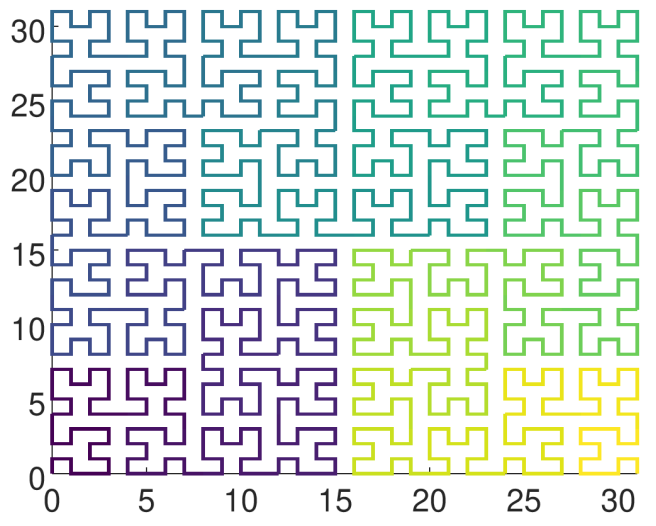

Figure 1: A standard Hilbert curve of order 5.

Listing 1 exemplifies a typical practice in implementations of standard Hilbert curves, which is to use an order $a$ leading to a square of edge length $2^{a}-1$ (i.e. 


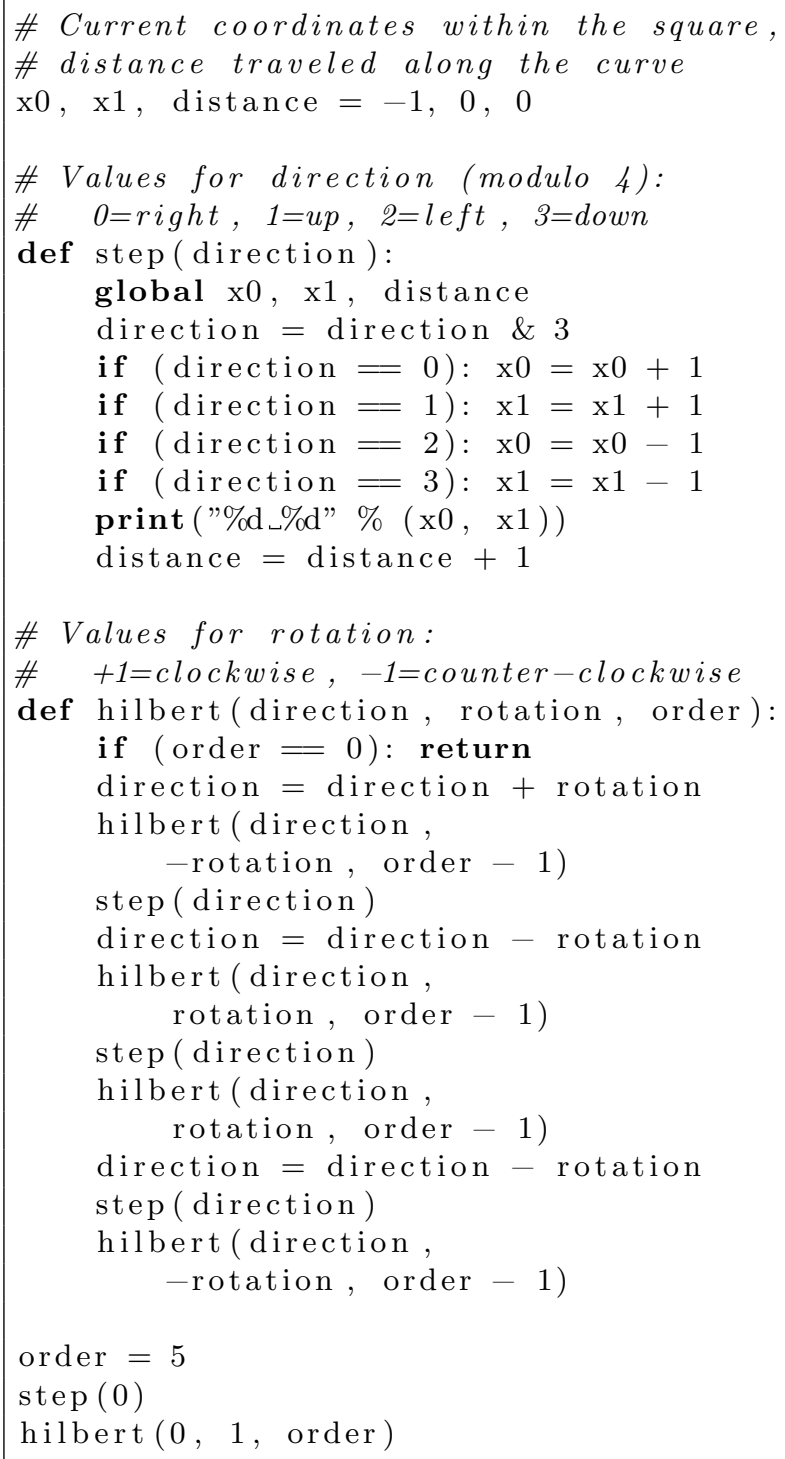

Listing 1: Generator program for a standard Hilbert curve of order 5 (adapted from Warren, 2013).

a power of two). Červený (2018) proposes an alternative approach that constructs generalized Hilbert curves over rectangles with edges of arbitrary lengths, using the desired lengths as input. Its implementation, which again is recursive, results in a recursion depth that we observed to be greater than the order of the equivalent standard Hilbert curve. That said, as this alternative approach can deal with edge lengths that are not a power of two, the concepts of order and depth cannot be compared directly. The intermediate coordinates and accumulated distance that result from both approaches are equal when the edge lengths are a power of two.

\section{Proposed Method}

In this section we describe the wave equation, how we solve it using SEM, and an explicit time integration scheme for the numerical solution. We also present an algorithm to reorder mesh-related data according to the generalized Hilbert curves described in Section 3.

\subsection{The Wave Equation}

The acoustic wave equation in 2D (Feynman et al., 2011) is a second-order PDE, that we restate as a first-order system over a domain $\Omega$ :

$$
\begin{gathered}
\dot{v}_{k}=\frac{1}{\rho} u_{, k}, \\
\frac{1}{K} \dot{u}=v_{k, k}+f .
\end{gathered}
$$

The unknowns are $u$ (pressure) and $v_{k}$. A single dot over a variable indicates a first time derivative. Variable $u$, a scalar, is continuous, whereas $v_{k}$, a vector with components for the $x_{0}$ and $x_{1}$ directions, is discontinuous. As for the remaining terms, $\rho$ is the density of the medium, $K$ is the compression modulus and $f$ is a source term. The values of $\rho$ and $K$ can vary with their location in the domain. The lower index,$k$ refers to the derivative with respect to direction $x_{k}$. We apply the Einstein convention with summation over double lower indices, i.e. in Eq. (2) there is a summation over index $k$.

The initial conditions are:

$$
u(\mathbf{x}, t=0)=v_{k}(\mathbf{x}, t=0)=0,
$$


for all locations $\mathbf{x}$ in the domain. There may also be boundary conditions to consider, such as Dirichlet and Neumann-type conditions (Butcher, 2008) which are not shown here.

We can regard the acoustic wave equation as a particular case of a broader notation that describes wave-type PDEs in 2D and 3D. For a scalar, continuous wave field $u$ with a secondary, discontinuous field $v_{k}$, we express this as:

$$
\begin{gathered}
M \dot{u}=-\left(B_{k l} v_{l}\right)_{, k}+D u+\sum_{s} y^{(s)}(t) \delta_{\mathbf{x}^{(s)}}, \\
\dot{v}_{k}=E_{k l} v_{l}+F_{k l} u_{, l},
\end{gathered}
$$

where $M, B_{k l}, D, E_{k l}$ and $F_{k l}$ are PDE coefficients assumed to be constant over time but variable in space, and $y^{(s)}$ is a time-dependent wave source applied at point $\mathbf{x}^{(s)}$. For the sake of a simpler presentation we assume that the continuous variable $u$ is a scalar and the discontinuous variable $v_{k}$ has two components. However, Eqs. (4) and (5) as well as the presented concepts can readily be extended to the more general case of a vector-valued continuous variable covering a wider range of applications such as elastic waves and Maxwell equations.

For the acoustic wave equation in $2 \mathrm{D}$ restated in Eqs. (2) and (1), $M$ and $D$ are scalar, whereas $B_{k l}$, $E_{k l}$ and $F_{k l}$ are matrices. This is $M=1 / K, B_{k k}=$ -1 and $F_{k k}=1 / \rho$ for $k=1,2$. $D$ and $E_{k l}$ are not used and therefore contain zeros.

\subsection{The Spectral Element Method}

The application of SEM requires converting the target PDEs into weak formulations, which for the generic Eq. (4) we find to be:

$$
\begin{aligned}
\int_{\Omega} M \dot{u} q d \Omega= & \int_{\Omega} B_{k l} v_{l} q, k d \Omega \\
& +\int_{\Omega} D u q d \Omega \\
& +\sum_{s} y^{(s)}(t) q\left(\mathbf{x}^{(s)}\right)
\end{aligned}
$$

where $q$ is an arbitrary test function that is smooth, in the sense that it is both continuous and piecewise differentiable.

We discretize Eqs. (6) and (5) in space using SEM. We subdivide the domain $\Omega$ into an unstructured mesh of non-overlapping triangular elements $\left(\Omega^{(e)}\right)$ covering the entire domain, where the upper index $e$ refers to the element count. All elements are based on a single reference element $\hat{\Omega}$ and described as $\mathbf{x}=\mathcal{F}^{(e)}(\hat{\mathbf{x}})$, with:

$$
x_{j}=x_{j}^{(e)}+J_{j \hat{j}}^{(e)} \hat{x}_{\hat{j}} \text { for } \hat{\mathbf{x}}=\left(\hat{x}_{j}\right) \in \hat{\Omega},
$$

where $\left(x_{j}^{(e)}\right)$ is an offset point, and $\left(J_{j \hat{j}}^{(e)}\right)$ is the Jacobian matrix describing the stretching and rotation of coordinates between $\hat{\Omega}$ and $\Omega^{(e)}$ for the transformation $\mathcal{F}^{(e)}$.

We represent the solution $u$ by its values $\left(U_{\mu}\right)$ at the global SEM nodes $\left(\mathbf{x}_{\mu}\right)$ of the mesh. On each element, we approximate the solution $u$ by a polynomial of a given order defined by a local basis function $\left(\hat{N}_{p}\right)$ at the reference element $\hat{\Omega}$. On the reference element $\hat{\Omega}$, the local nodes $\hat{\mathbf{x}}_{q}$ and local basis function $\left(\hat{N}_{p}\right)$ form a dual system:

$$
\hat{N}_{p}\left(\hat{\mathbf{x}}_{q}\right)=\delta_{p q}
$$

An important aspect of SEM is that the local nodes used to approximate the solution at each element are also quadrature points for numerical integration (Komatitsch and Vilotte, 1998). In our proposed implementation, we use local nodes over variable-sized triangles and Appell polynomials as basis functions as described by Blyth and Pozrikidis (2006). Resuming the previous discussion, we approximate the solution at $\mathbf{x} \in \Omega^{(e)}$ as:

$$
u(\mathbf{x})=\sum_{p} \hat{N}_{p}(\hat{\mathbf{x}}) U_{\mu(p, e)} \text { with } \mathbf{x}=\mathcal{F}^{(e)}(\hat{\mathbf{x}})
$$

where the index $\mu(p, e)$ maps the local SEM node $\hat{\mathbf{x}}_{p}$ to the corresponding global node:

$$
\mathbf{x}_{\mu(p, e)}=\mathcal{F}^{(e)}\left(\hat{\mathbf{x}}_{p}\right)
$$


Given the condition at Eq. (8), it follows that $U_{\mu}$ is the value of $u$ at global node $\mathbf{x}_{\mu}$. The derivative of $u$ at element $\Omega^{(e)}$ is then given as:

$$
\begin{array}{r}
u_{, j}(\mathbf{x})=\sum_{p, \hat{j}} K_{j \hat{j}}^{(e)} \hat{N}_{p, \hat{j}}(\hat{\mathbf{x}}) U_{\mu(p, e)} \\
\text { for } \mathbf{x}=\mathcal{F}^{(e)}(\hat{\mathbf{x}}) \in \Omega^{(e)},
\end{array}
$$

where $\left(K_{j \hat{j}}^{(e)}\right)$ is the inverse matrix of $\left(J_{j \hat{j}}^{(e)}\right)$.

We store the values of discontinuous function $v_{k}$ at the local SEM nodes $\hat{\mathbf{x}}_{p}$ in element $e$ with values $V_{k p}^{(e)}$. Assuming that coefficients $E$ and $F$ are constant in each element with values $E^{(e)}$ and $F^{(e)}$, respectively, and applying the derivative $u_{, j}$ (x) found at Eq. (11), we discretize Eq. (5) at each local node in $\Omega^{(e)}$ as:

$$
\begin{aligned}
\dot{V}_{k p}^{(e)}= & \sum_{l} E_{k l}^{(e)} V_{l p}^{(e)} \\
& +\sum_{q, j, \hat{j}} F_{k j}^{(e)} K_{j \hat{j}}^{(e)} \hat{N}_{q, \hat{j}}\left(\hat{\mathbf{x}}_{p}\right) U_{\mu(q, e)} .
\end{aligned}
$$

Proceeding to Eq. (6) we employ the fact that, with SEM, the local nodes of each element double as nodes for a numerical integration scheme. On the reference element, we express this as:

$$
\int_{\hat{\Omega}} \hat{f} d \hat{x} \approx \sum_{q} \hat{\omega}_{q} \hat{f}\left(\hat{\mathbf{x}}_{q}\right)
$$

for any function $\hat{f}$ defined on $\hat{\Omega}$, where $\hat{\omega}_{q}$ are the integration weights. For a function $f$ defined on $\Omega$, we have:

$$
\int_{\Omega} f d x=\sum_{e} \int_{\Omega^{(e)}} f d x \approx \sum_{e} \mathcal{J}^{(e)} \sum_{q} \hat{\omega}_{q} f_{q e}
$$

with $f_{q e}=f\left(\mathbf{x}_{\mu(q, e)}\right)$ and $\mathcal{J}^{(e)}=\operatorname{det}\left(J_{j \hat{j}}^{(e)}\right)$, where $\mathcal{J}^{(e)}$ is dependent on the element but independent of the integration node. Assuming that the PDE coefficients are constant on each element, we rewrite Eq. (6) as:

$$
\dot{U}_{\nu}=\bar{M}_{\nu}^{-1}\left(R_{\nu}+\bar{D}_{\nu} U_{\nu}+y_{\nu}(t)\right)
$$

where for $A=M, D$ :

$$
\bar{A}_{\nu}=\sum_{e, q ; \nu=\mu(q, e)} A^{(e)} \mathcal{J}^{(e)} \hat{\omega}_{q}
$$

and:

$$
\begin{gathered}
R_{\nu}=\sum_{e, p, q ; \nu=\mu(p, e)} \mathcal{J}^{(e)} \hat{\omega}_{q} \sum_{k, j, \hat{j}} B_{k j}^{(e)} V_{k q}^{(e)} K_{j \hat{j}}^{(e)} \hat{N}_{p, \hat{j}}\left(\hat{\mathbf{x}}_{q}\right), \\
y_{\nu}(t)=y^{(s)}(t) \text { for } \mathbf{x}^{(s)}=\mathbf{x}_{\nu} .
\end{gathered}
$$

Notice that calculating the rate of change for discontinuous variable $V_{k p}^{(e)}$ in Eq. (12) can be performed independently for each element $e$, but requires the gather operation $U_{\mu(q, e)}$ to collect values of continuous variable $U_{\nu}$ at the global SEM nodes corresponding to the local SEM nodes at each element. In contrast, the rate of change of continuous variable $U_{\nu}$ in Eq. (15) requires accumulating results of an elementby-element calculation at global SEM nodes. This operation creates a race condition when parallelized, as several elements can share global SEM nodes.

\subsubsection{Time Integration}

The explicit time integration scheme starts at $n=0$, with known $V_{k p}^{(e, n=0)}=0$ and $U_{\nu}^{(n=0)}=0$. Let $t^{(n)}=$ $t^{(0)}+n \cdot h$, where $h$ is the time step size. With Eqs. (15) and (12) describing how $U$ and $V_{k}$ change over time, we use the Heun method (Heun, 1900) to update these variables at each time step. The predictor step is:

$$
\begin{gathered}
\tilde{U}_{\nu}^{(n+1)}=U_{\nu}^{(n)}+h \cdot \dot{U}_{\nu}^{(n)}, \\
\tilde{V}_{k p}^{(e, n+1)}=V_{k p}^{(e, n)}+h \cdot \dot{V}_{k p}^{(e, n)},
\end{gathered}
$$

followed by the corrector step:

$$
\begin{aligned}
U_{\nu}^{(n+1)} & =U_{\nu}^{(n)}+\frac{h}{2} \cdot\left(\dot{U}_{\nu}^{(n)}+\dot{\tilde{U}}_{\nu}^{(n+1)}\right), \\
V_{k p}^{(e, n+1)} & =V_{k p}^{(e, n)}+\frac{h}{2} \cdot\left(\dot{V}_{k p}^{(e, n)}+\dot{\tilde{V}}_{k p}^{(e, n+1)}\right),
\end{aligned}
$$

where $\dot{\tilde{U}}_{\nu}^{(n+1)}$ and $\dot{\tilde{V}}_{k p}^{(e, n+1)}$ are the results of Eqs. (15) and (12) evaluated using predictors $\tilde{U}_{\nu}^{(n+1)}$ and 
$\tilde{V}_{k p}^{(e, n+1)}$ as input. We can use higher-order methods such as fourth-order Runge-Kutta (Runge, 1895; Kutta, 1901) for improved accuracy, at the cost of higher processing requirements.

\subsection{Memory Reordering with Gener- alized Hilbert Curves}

To leverage the data locality provided by the spatial proximity features of generalized Hilbert curves, we propose the following memory reordering algorithm for SEM-based equation solvers:

1. Load the mesh into memory, and compute the centroids of all elements;

2. Let $w_{m}$ and $h_{m}$ be the width and the height of the mesh, $r$ the ratio $w_{m} / h_{m}$, and $n_{e}$ the number of elements. Define a bounding box with width $w_{b}=\sqrt{n_{e}}$ and height $h_{b}=\sqrt{n_{e}} / r$, made of subrectangles of width $w_{s}=w_{b} / w_{m}$ and height $h_{s}=h_{b} / h_{m}$. Using the technique proposed by Červený (2018), generate a Hilbert curve over that bounding box, and store the $\left(x_{0}, x_{1}\right)$-coordinates of the intermediate steps of the curve;

3. Let $\left(x_{m 0}, x_{m 1}\right)$ be the minimum coordinates of the mesh in the horizontal and vertical axes. Given the centroid of an element, let $\left(x_{c 0}, x_{c 1}\right)$ be its coordinates in the horizontal and vertical axes. Traverse the intermediate steps of the Hilbert curve stored previously, and, at each step, check which subrectangle of the bounding box the step is located in; check the elements of the mesh whose centroids map to that subrectangle (i.e. the elements for which the coordinates $\left(x_{c 0}-x_{m 0}\right) \cdot w_{s}$ and $\left(x_{c 1}-x_{m 1}\right) \cdot h_{s}$ are inside the subrectangle), and accumulate those elements in a list $L$. Each element must be stored in $L$ only once;

4. After traversing all elements, $L$ contains all elements and their order closely resembles that of the steps of the Hilbert curve. Now we must relabel elements and rearrange related data in memory, according to the optimized order contained in $L$.

After executing this algorithm, sequential traversals of mesh elements closely match the steps of the Hilbert curve, resulting in increased data locality and memory efficiency. We apply this reordering to all data structures that are traversed element-by-element.

We employ the algorithm in the same way with structured or unstructured meshes. With structured meshes, we expect regular patterns in the number of elements found at intermediate steps of the Hilbert curve. With unstructured meshes, however, because of variable geometric complexity, we must not assume any uniformity in how many elements each step of the curve encompasses: the steps contained in certain regions may match many elements, whereas steps contained in other regions may match only a few elements (or possibly none), in an unpredictable fashion. An alternative to change the number of elements found at each step of the curve is to adjust the width and height of the bounding box while honoring the proportion $w_{b} / h_{b}=r$ (for instance, using $w_{b}=\sqrt{n_{e}} \cdot r$ and $\left.h_{b}=\sqrt{n_{e}}\right)$.

We can achieve additional memory efficiency by also reordering global nodes. The proposed procedure, based on the Cuthill-McKee algorithm (Cuthill and McKee, 1969), follows:

1. Traverse all elements; at each element, accumulate all of its nodes in a list $N^{(e)}$. A node must not be stored in $N^{(e)}$ if it has been processed in a previous element;

2. Each node in $N^{(e)}$ has a degree, i.e. the number of connections that the node has to other nodes. Sort the nodes in $N^{(e)}$ by ascending degree; relabel the nodes and rearrange related data in memory, according to the optimized order contained in $N^{(e)}$.

We apply this reordering strategy to all data structures that are traversed node-by-node. 


\section{Software Implementation}

We developed a 2D acoustic wave propagation simulator in the esys-escript tool, using the concepts and equations discussed in Section 4.2 and the Heun scheme described in Section 4.2.1. We built its central functionality - the explicit time integration scheme - in C++, leveraging OpenMP (OpenMP, 2021) for multithreading.

In describing the implementation of the time integration scheme, we assess the computational effort of traversing data structures element-by-element at each time step. To that end, we analyze Eqs. (12) and (15), which describe how variables $U$ and $V_{k}$ change over time. The layout of their terms in memory (see Figure 2) is important regarding the behavior of the processor cache:

- PDE coefficients $\bar{M}_{\nu}$ and $\bar{D}_{\nu}$ and continuous variable $U_{\nu}$ are arrays of scalars, with each item in the arrays corresponding to a global node $\nu$. We store them in the order of continuous labeling of the global nodes;

- We define PDE coefficients $B_{k l}^{(e)}, E_{k l}^{(e)}$ and $F_{k l}^{(e)}$ at each element $e$ as matrices, where $k$ is the row and $l$ is the column. We store them in the order of continuous labeling of the elements, following a Fortran standard storing rows continuously in memory;

- We define discontinuous variable $V_{k p}^{(e)}$ at each local node $p$ of each element $e$, with two components for directions $x_{0}$ and $x_{1}$ indexed by $k$. We store its instances in the order of continuous labeling of the elements.

We examine Eq. (12) for the change in $V_{k}$. The pseudo-code for its first term, $\sum_{l} E_{k l}^{(e)} V_{l p}^{(e)}$, is in the first code block of Algorithm 1. The main factors for the computational complexity of this term are the number of elements and the number of nodes per element, which stems from the polynomial approximation order. The remainder of this discussion considers the case where the discontinuous variable $v_{k}$ has two components, therefore $k$ varies from 1 to 2 . Since $E_{k l}^{(e)}$ is a square matrix in this case, $l$ behaves the same as

\begin{tabular}{|c|c|c|c|c|c|c|c|c|}
\hline & & & $\bar{M}_{1}$ & $\bar{M}_{2}$ & $\cdots$ & & & \\
\hline & & & & bal no & des & & & \\
\hline$B_{1,1}^{(1)}$ & $B_{1,2}^{(1)}$ & $B_{2,1}^{(1)}$ & $B_{2,2}^{(1)}$ & $B_{1,1}^{(2)}$ & $B_{1,2}^{(2)}$ & $B_{2,1}^{(2)}$ & $B_{2,2}^{(2)}$ & $\ldots$ \\
\hline & elem & ent 1 & & & elem & ent 2 & & \\
\hline$V_{1,1}^{(1)}$ & $V_{2,1}^{(1)}$ & $\ldots$ & $V_{1, p}^{(1)}$ & $V_{2, p}^{(1)}$ & $V_{1,1}^{(2)}$ & $V_{2,1}^{(2)}$ & $\ldots$ & $\ldots$ \\
\hline \begin{tabular}{|l|} 
local \\
\end{tabular} & ode 1 & & local r & node $p$ & & & & \\
\hline
\end{tabular}

Figure 2: Memory layout of equation terms for global node-based values $\bar{M}_{\nu}$, element-based values $B_{k l}^{(e)}$ and local node-based values $V_{k p}^{(e)} . \bar{D}_{\nu}$ and $U_{\nu}$ have the same layout as $\bar{M}_{\nu}$, and $E_{k l}^{(e)}$ and $F_{k l}^{(e)}$ have the same layout as $B_{k l}^{(e)}$.

$k$ and also varies from 1 to 2 . This assumption of $k$ and $l$ allows to calculate the initial term of $\dot{V}_{k p}^{(e)}$ in a single statement without looping over $k$ and $l$.

The second term, a summation over $q, j$ and $\hat{j}$, is more expensive than the first. As shown in the pseudo-code, the $\dot{V}_{k p}^{(e)}$ accumulation step requires three nested loops where we introduce two precalculations to eliminate redundant calculations. For the first precalculation of array $F K$, as $k$ varies from 1 to 2 and $F_{k j}^{(e)}$ and $K_{j \hat{j}}^{(e)}$ are square matrices, $j$ and $\hat{j}$ vary from 1 to 2 . Assuming $F_{k j}^{(e)}$ to be equal for all nodes within an element, we compute the values of $F_{k j}^{(e)} K_{j \hat{j}}^{(e)}$ in a single statement eliminating iterations over directions $j$ and $\hat{j}$; we store them in the $F K$ array, which is independent of local node $p$ and integration point $q$ and reduces memory accesses and arithmetic operations in the accumulation step later. Moving on to the second precalculation, we need to access the value of solution $\left(U_{\mu}\right)$ at global node $\mu(q, e)$, a gather operation. We cache the values of $U_{\mu(q, e)}$ in the $U_{2}$ array to improve data locality and reduce potential repeated cache line fetches. Finally, at the $\dot{V}_{k p}^{(e)}$ accumulation step, the value of $q$ is equal to the number of nodes per element; given the assumptions for $j$ and 


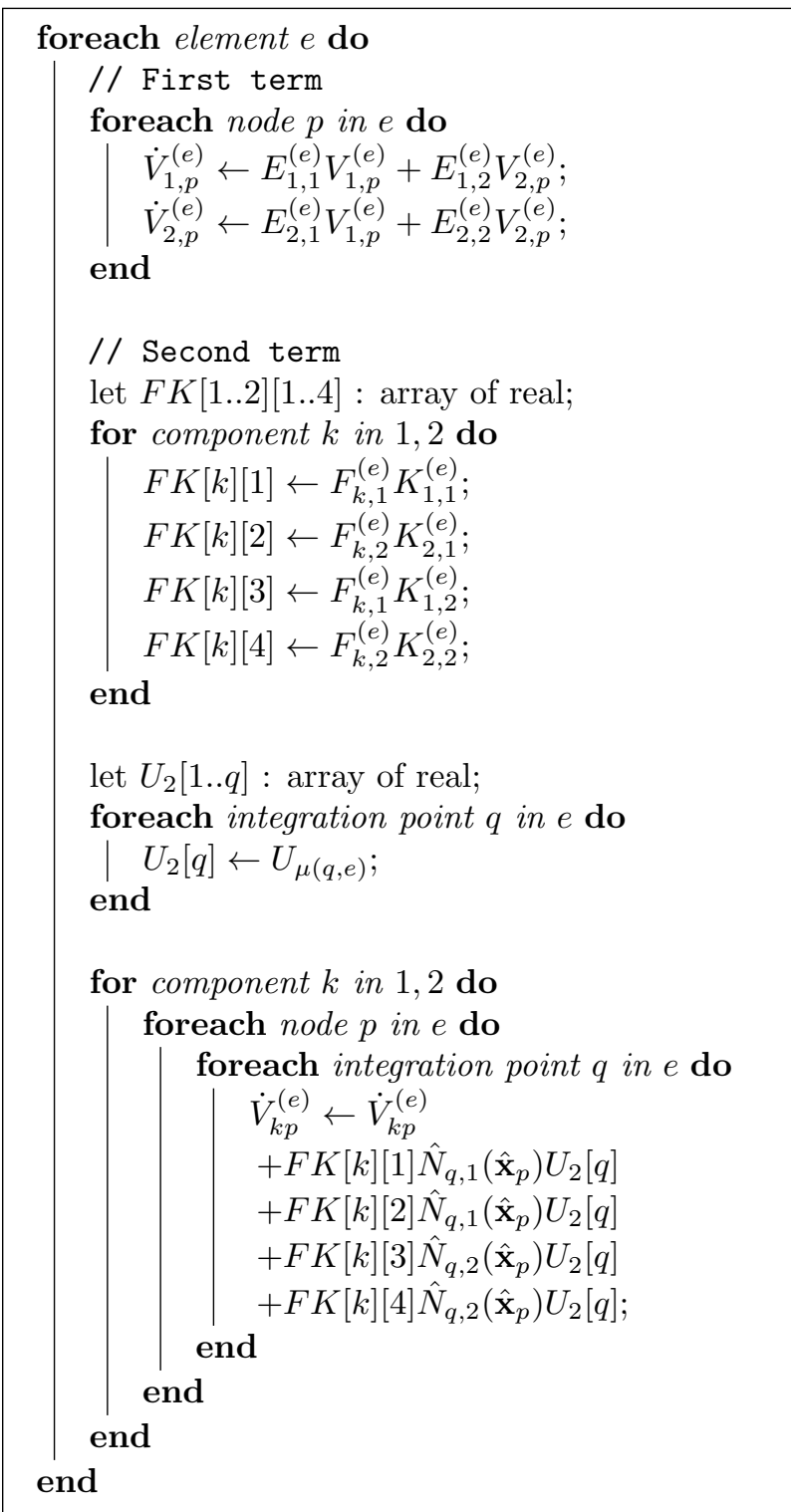

Algorithm 1: Calculation of $\dot{V}_{k p}^{(e)}$ by Eq. (12) for a discontinuous variable $v_{k}$ with two components.

$\hat{j}$, we write the accumulation expression in a single statement without looping over $j$ and $\hat{j}$. Since both terms of Eq. (12) do not affect data of elements other than the current element, Algorithm 1 is straightfor- ward to parallelize by distributing elements among separate threads.

Proceeding to Eq. (15), which describes how $U$ changes over time, we start by examining its second term, $\bar{M}_{\nu}^{-1} \bar{D}_{\nu} U_{\nu}$, in Algorithm 2. We precalculate $Y_{\nu}=\bar{M}_{\nu}^{-1} \bar{D}_{\nu}$ for all global nodes before time integration and use it to initialize $\dot{U}_{\nu}$. This term of Eq. (15) is straightforward to parallelize, as it does not affect the data of global nodes other than the current node.

The first term of Eq. (15), $\bar{M}_{\nu}^{-1} R_{\nu}$, involves more computational work. We obtain $R_{\nu}$ by summation over all elements $e$, local nodes $p$ and integration points $q$ with $\nu=\mu(p, e)$. We must loop over the elements and their local nodes, adding contributions to the respective global nodes. Analogously to $F_{k j}^{(e)} K_{j \hat{j}}^{(e)}$ in Algorithm 1, there is a precalculation section where we compute the values of $B_{k j}^{(e)} K_{j \hat{j}}^{(e)}$; we store them in the $B K$ array, which is independent of $p$ and $q$ and eliminates redundant arithmetic operations in the accumulation step later.

The accumulation of contributions to add to $\dot{U}_{\nu=\mu(p, e)}$ occurs in the val variable at the nested forloops that traverse $k$ and $q$, and we perform it in a single statement without loops, exploiting the assumptions on $j$ and $\hat{j}$ counts. The update of $\dot{U}_{\nu}$ with the accumulated value stored at val is a gather operation, as the solution value for global node $\nu=\mu(p, e)$ receives contributions of all local nodes within elements that map to that global node, and a global node may be shared among neighboring elements.

Since local nodes of several elements may share a single global node, we must be careful when updating $\dot{U}_{\nu}$ and processing multiple elements simultaneously. If any two elements that share a global node $\nu$ update $\dot{U}_{\nu}$ concurrently, race conditions may occur. To avoid that situation, we use element coloring (Davies et al., 2004). When we first construct the mesh, we regard the elements of the mesh as vertices of a graph. We consider any two elements sharing a global node to be connected by an edge in the graph, and use a greedy vertex-coloring algorithm to assign different colors to any two elements sharing a global node. This allows processing elements of identical color simultaneously without mishandling operations over $\dot{U}_{\nu}$. That way, whereas we must traverse the available colors sequen- 


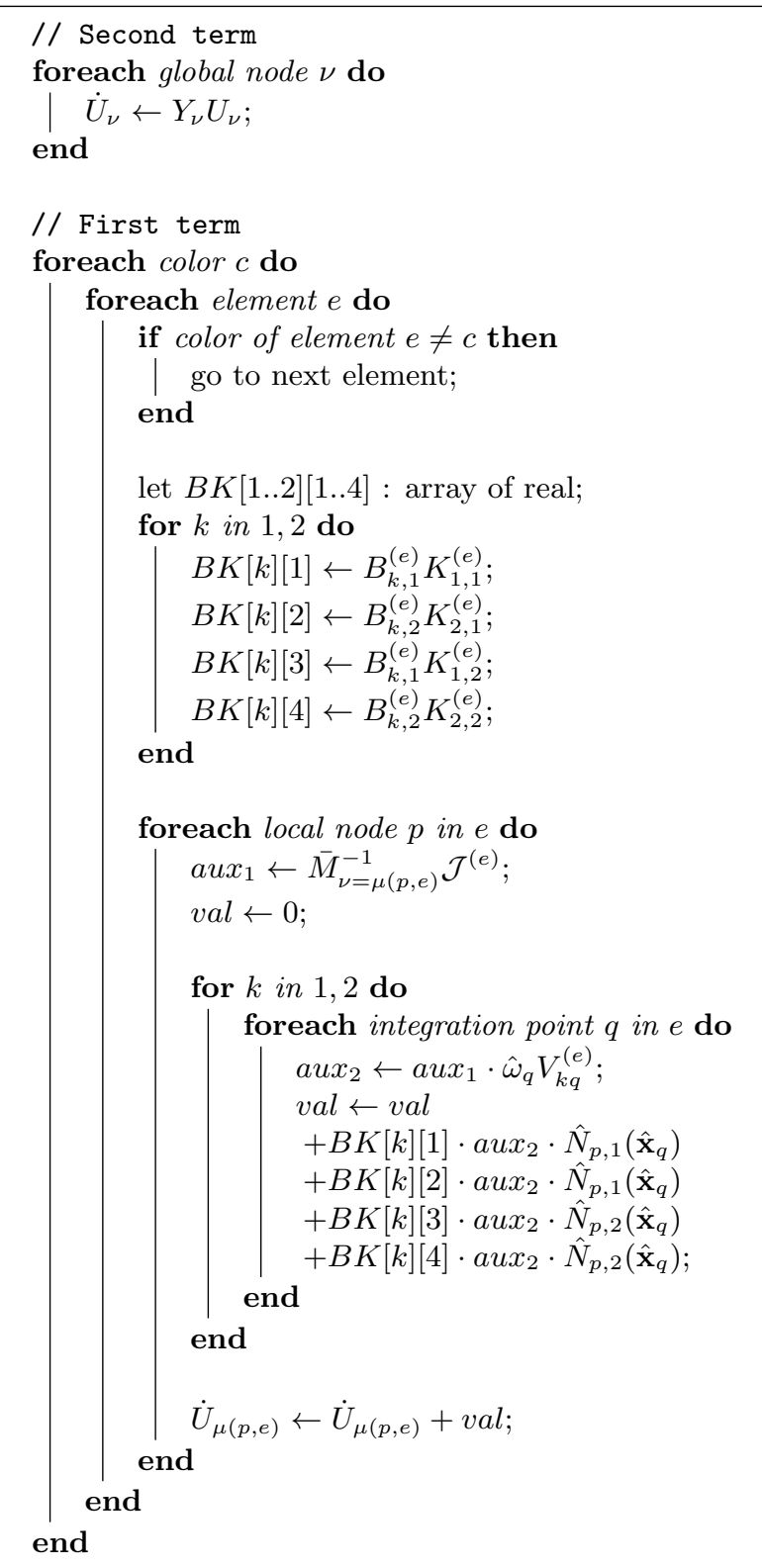

Algorithm 2: Calculation of $\dot{U}_{\nu}$ via the second and first terms of Eq. (15) with precalculated $Y_{\nu}=\bar{M}_{\nu}^{-1} \bar{D}_{\nu}$.

tially in the $c$ for-loop of Algorithm 2, we can safely parallelize the remaining work at the $e$ for-loop that

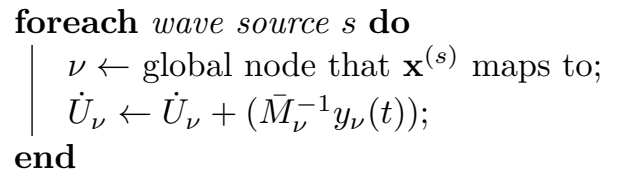

foreach wave source $s$ do

$\nu \leftarrow$ global node that $\mathbf{x}^{(s)}$ maps to; $\dot{U}_{\nu} \leftarrow \dot{U}_{\nu}+\left(\bar{M}_{\nu}^{-1} y_{\nu}(t)\right)$

end

Algorithm 3: Calculation of the source term, which is the third term of Eq. (15).

follows. The number of colors is contingent on mesh complexity.

The third and final term in Eq. (15), $\bar{M}_{\nu}^{-1} y_{\nu}(t)$, is simple. As listed in Algorithm 3, we need only to traverse the global nodes in the domain that contain wave sources and accumulate their contributions into $\dot{U}_{\nu}$. The accumulation step for $\dot{U}_{\nu}$ assumes that Algorithm 2 was executed beforehand. Typically the number of point sources is small and parallelizing this segment is not worthwhile.

We need to execute the steps in Algorithms 1 through 3 to compute Eqs. (12) and (15) at every step of the time integration scheme. As per Heun's method, we compute those equations twice at every time step, first for the predictor phase and then for the corrector phase.

\section{Results and Discussion}

In this section, we assess the execution speed of our proposed implementation under a number of scenarios. We follow by demonstrating the application of our acoustic wave simulator to a complex, realistic domain.

\subsection{Performance Benchmarks}

We executed two benchmarks of our simulator using meshes with linear elements of synthetic domains generated with the mesh generator tool Gmsh (Geuzaine and Remacle, 2009). As Gmsh does not generate SEM meshes, we added SEM nodes to the meshes in memory using the locations described by Blyth and Pozrikidis (2006). The simulator supports four memory reordering strategies for mesh-related data, described next. 
No Strategy: In this strategy, we simply store element and node-related data in the same order as obtained from the original source. Generally speaking, we cannot make any assumptions on the data order. However, this should not necessarily be considered as a random ordering.

Node Connectivity Strategy: This strategy is very similar to the Cuthill-McKee algorithm (Cuthill and McKee, 1969). We pick the node with the smallest number of connections in the mesh and proceed to the node with the smallest number of connections that is connected to that node; we repeat the process, avoiding previously visited nodes, until all nodes have been traversed. At each node, we accumulate the element that the node belongs to in a list and the node itself in another list, avoiding duplicate items. Finally, we relabel the elements and nodes and reorder related data in memory following the order contained in the lists.

Node Distance Strategy: This strategy closely resembles the node connectivity strategy, the only difference being that we pick the node closest to a reference point and proceed to the node closest to the reference point that is connected to that node. We repeat this process, avoiding previously visited nodes, until all nodes have been traversed. In our experiments, we always positioned the chosen reference point in the lower-left corner of the domain.

Hilbert SFC Strategy: In this strategy, we apply the algorithm described in Section 4.3.

In our benchmarks, after loading meshes from local files and adding SEM nodes, memory reordering made the mesh preparation process take between $15 \%$ and $20 \%$ longer than performing no reordering. In the following discussions, the labels "None", "Conn.", "Dist." and "SFC" refer to the application of no strategy, node connectivity, node distance and Hilbert curve memory reordering strategy, respectively.

\subsubsection{Single-Layered Domain}

We divided the first benchmark into three parts. In the first part, we simulated the propagation of an acoustic wave in a single-layered domain to assess the average CPU time required to compute a single time step of the wave. The medium density, compression modulus and wave speed were constant throughout the domain. We used a GNU/Linux system running CentOS 6.5 64-bit equipped with an Intel Xeon E5-2698 v3 CPU, with sixteen physical cores and two hardware threads per core, for 32 threads in total. All cores ran at $2.3 \mathrm{GHz}$ and total cache size was 40 MB. We used six different thread counts between 1 and 32, and three different mesh granularities: 250 thousand, 500 thousand and 1 million elements. Element coloring resulted in 11 colors (first and third meshes) and 12 colors (second mesh). The domain was 2000 units wide and 1000 units deep, resulting in SFC depths of 16 (first mesh) and 18 (second and third meshes). Each mesh, although unstructured, had elements of largely homogeneous edge size. We used a polynomial order of 5, resulting in 21 local nodes per element. The execution results are listed in Table 1 and plotted in Figure 3.

The CPU times in Table 1 and Figure 3 show that the "SFC" strategy was always the fastest option. It was most advantageous when compared to the "None" strategy, especially when using a larger number of threads. We highlight the fact that the "Conn." and "Dist." strategies were sometimes slower than the "None" strategy, as seen in the results for one and two threads. Therefore, when developing reordering strategies, we must consider that performance may actually decline in specific cases. Another observation from Figure 3 is that the speedup of SFCbased ordering in relation to the fastest serial time for each mesh granularity was always higher than all other strategies.

In the second part of the first benchmark, we ran simulations with several polynomial orders and mesh granularities of the single-layered domain using all available 32 threads. The results are shown in Table 2 and plotted in Figure 4. The "SFC" strategy retained the best performance, running between $14.8 \%$ (one million elements, order 7) and $25.1 \%$ (250 thousand elements, order 5) faster than the "None" strategy. However we must point that, as the mesh granularity increased, the higher data volume per element did not benefit from using SFC-based ordering as much.

The third and final part of the first benchmark mea- 


\begin{tabular}{|c|c|c|c|c|c|c|c|c|c|c|c|c|}
\hline \multirow[b]{4}{*}{ Threads } & \multicolumn{12}{|c|}{ Average CPU time (s) per time step } \\
\hline & \multicolumn{4}{|c|}{ 250k-element domain } & \multicolumn{4}{|c|}{ 500k-element domain } & \multicolumn{4}{|c|}{ 1M-element domain } \\
\hline & \multicolumn{4}{|c|}{ Reordering strategy } & \multicolumn{4}{|c|}{ Reordering strategy } & \multicolumn{4}{|c|}{ Reordering strategy } \\
\hline & None & Conn. & Dist. & $\mathrm{SFC}$ & None & Conn. & Dist. & SFC & None & Conn. & Dist. & $\mathrm{SFC}$ \\
\hline 1 & 5.292 & 5.786 & 5.773 & 4.884 & 10.977 & 11.813 & 11.782 & 10.016 & 22.847 & 24.141 & 24.101 & 20.415 \\
\hline 2 & 4.348 & 4.454 & 4.449 & 4.015 & 8.982 & 9.127 & 9.100 & 8.225 & 18.411 & 18.425 & 18.382 & 16.517 \\
\hline 4 & 2.368 & 2.283 & 2.275 & 2.054 & 4.905 & 4.711 & 4.700 & 4.276 & 10.041 & 9.542 & 9.522 & 8.639 \\
\hline 8 & 1.307 & 1.176 & 1.185 & 1.066 & 2.704 & 2.474 & 2.468 & 2.235 & 5.519 & 5.005 & 5.006 & 4.527 \\
\hline 16 & 0.721 & 0.628 & 0.627 & 0.570 & 1.490 & 1.333 & 1.333 & 1.228 & 3.057 & 2.696 & 2.714 & 2.483 \\
\hline 32 & 0.436 & 0.359 & 0.354 & 0.327 & 0.867 & 0.772 & 0.775 & 0.697 & 1.736 & 1.576 & 1.568 & 1.465 \\
\hline
\end{tabular}

Table 1: Benchmark 1 on Intel Xeon: Average CPU time to compute a single time step in a 32-thread system for several thread counts and mesh granularities of a single-layered domain using polynomial order 5 .
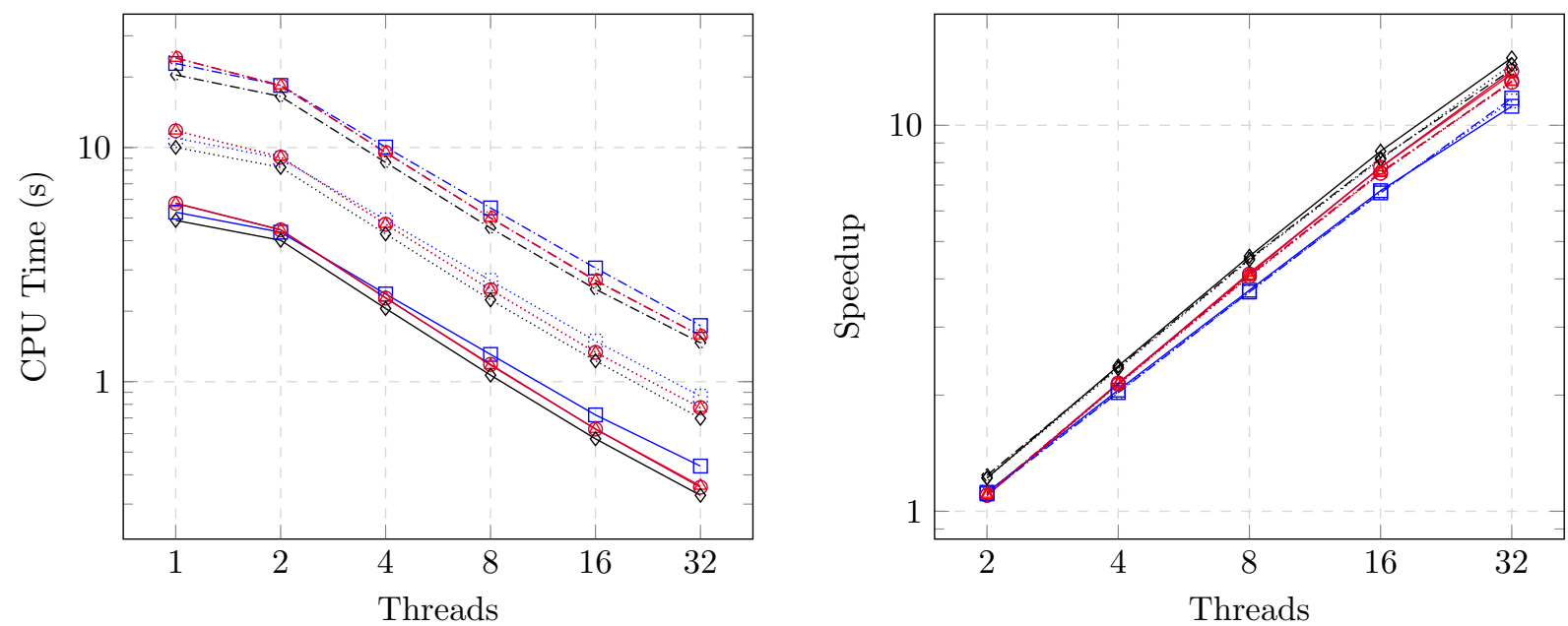

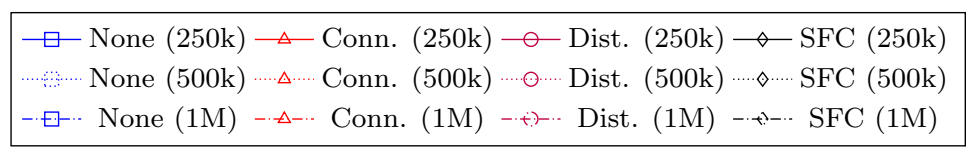

Figure 3: Benchmark 1 on Intel Xeon: Average CPU time to compute a single time step (left) and average speedup in relation to the fastest serial time (right) for several thread counts and mesh granularities of a single-layered domain using polynomial order 5 . Values are shown in Table 1 . Time, speedup and threads are in logarithmic scale.

sured the number of last-level cache (LLC) misses and the percentage of stalled slots in the memory pipeline (SSMP) on the single-layered domain. This time we used a GNU/Linux system running Ubuntu 18.04.5 LTS equipped with an Intel i7-7500U CPU, with two physical cores and two hardware threads per core, for four threads in total. All cores ran at $3.5 \mathrm{GHz}$ and to- tal cache size was $4 \mathrm{MB}$. Polynomial order was fixed at 5. We made measurements using Intel VTune Profiler (2021), and they are shown in Table 3 and Figure 5. SFC-based ordering once again yielded the lowest compute time, with the corresponding number of LLC misses and SSMP percentage significantly lower than the other strategies. It also stands out that, under 


\begin{tabular}{|c|c|c|c|c|c|c|c|c|c|c|c|c|}
\hline \multirow[b]{4}{*}{ Order } & \multicolumn{12}{|c|}{$\begin{array}{c}\text { Average CPU time (s) per time step, } \\
\text { percentage of time in relation to "None" reordering strategy }\end{array}$} \\
\hline & \multicolumn{4}{|c|}{ 250k-element domain } & \multicolumn{4}{|c|}{ 500k-element domain } & \multicolumn{4}{|c|}{ 1M-element domain } \\
\hline & \multicolumn{4}{|c|}{ Reordering strategy } & \multicolumn{4}{|c|}{ Reordering strategy } & \multicolumn{4}{|c|}{ Reordering strategy } \\
\hline & None & Conn. & Dist. & $\mathrm{SFC}$ & None & Conn. & Dist. & $\mathrm{SFC}$ & None & Conn. & Dist. & $\mathrm{SFC}$ \\
\hline 5 & $\begin{array}{c}0.436 \\
-\end{array}$ & $\begin{array}{l}0.359 \\
82.4 \%\end{array}$ & $\begin{array}{l}0.354 \\
81.1 \%\end{array}$ & $\begin{array}{l}0.327 \\
74.9 \%\end{array}$ & $\begin{array}{c}0.867 \\
-\end{array}$ & $\begin{array}{l}0.772 \\
89.1 \%\end{array}$ & $\begin{array}{l}0.775 \\
89.4 \%\end{array}$ & $\begin{array}{l}0.697, \\
80.5 \%\end{array}$ & $\begin{array}{c}1.736, \\
-\end{array}$ & $\begin{array}{l}1.576, \\
90.8 \%\end{array}$ & $\begin{array}{l}1.568, \\
90.4 \%\end{array}$ & $\begin{array}{l}1.465, \\
84.4 \%\end{array}$ \\
\hline 6 & $\begin{array}{c}0.644, \\
-\end{array}$ & $\begin{array}{l}0.533 \\
82.8 \%\end{array}$ & $\begin{array}{l}0.531 \\
82.4 \%\end{array}$ & $\begin{array}{l}0.514 \\
79.9 \%\end{array}$ & $\begin{array}{c}1.285 \\
-\end{array}$ & $\begin{array}{l}1.117 \\
86.9 \%\end{array}$ & $\begin{array}{l}1.119 \\
87.1 \%\end{array}$ & $\begin{array}{l}1.080 \\
84.1 \%\end{array}$ & $\begin{array}{c}2.646, \\
-\end{array}$ & $\begin{array}{l}2.344 \\
88.6 \%\end{array}$ & $\begin{array}{l}2.355 \\
89.0 \%\end{array}$ & $\begin{array}{l}2.238 \\
84.6 \%\end{array}$ \\
\hline 7 & $\begin{array}{c}0.970 \\
-\end{array}$ & $\begin{array}{l}0.804 \\
82.9 \%\end{array}$ & $\begin{array}{l}0.809 \\
83.4 \%\end{array}$ & $\begin{array}{l}0.775 \\
79.8 \%\end{array}$ & $\begin{array}{c}1.967 \\
-\end{array}$ & $\begin{array}{l}1.681 \\
85.4 \%\end{array}$ & $\begin{array}{l}1.680 \\
85.4 \%\end{array}$ & $\begin{array}{l}1.618, \\
82.2 \%\end{array}$ & $\begin{array}{c}4.010, \\
-\end{array}$ & $\begin{array}{l}3.541 \\
88.3 \%\end{array}$ & $\begin{array}{l}3.575 \\
89.2 \%\end{array}$ & $\begin{array}{l}3.414, \\
85.2 \%\end{array}$ \\
\hline
\end{tabular}

Table 2: Benchmark 1 on Intel Xeon: Average CPU time to compute a single time step and percentage of time in relation to "None" reordering strategy, for several polynomial orders and mesh granularities of a single-layered domain using 32 threads.

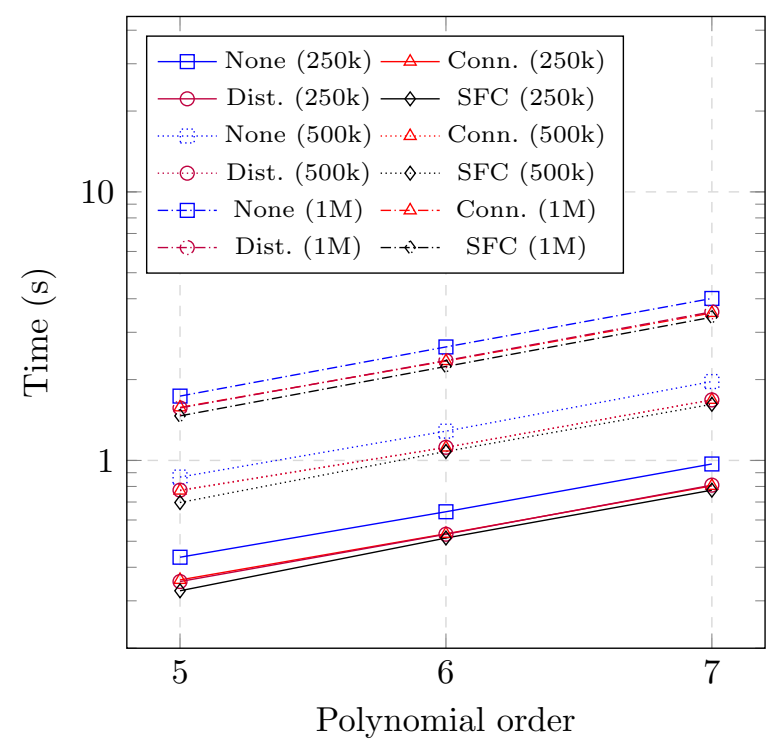

Figure 4: Benchmark 1 on Intel Xeon: Average CPU time to compute a single time step for several polynomial orders and mesh granularities of a single-layered domain using 32 threads. Values are shown in Table 2. Time is in logarithmic scale.

the "Conn." and "Dist." strategies, LLC misses were noticeably higher than those of the "None" strategy, but all SSMP percentages were slightly lower and per- formance was always better.

\begin{tabular}{|c|c|c|c|c|}
\hline \multirow[b]{3}{*}{$\begin{array}{l}\text { Domain } \\
\text { elements }\end{array}$} & \multicolumn{4}{|c|}{$\begin{array}{l}\text { Average CPU time (s), } \\
\text { LLC misses (millions), } \\
\text { SSMP percentage }\end{array}$} \\
\hline & \multicolumn{4}{|c|}{ Reordering strategy } \\
\hline & None & Conn. & Dist. & $\mathrm{SFC}$ \\
\hline $250 \mathrm{k}$ & $\begin{array}{c}1.445 \\
1021.03 \\
14.9 \%\end{array}$ & $\begin{array}{c}1.330 \\
1489.78 \\
14.7 \%\end{array}$ & $\begin{array}{c}1.329 \\
1453.06 \\
14.4 \%\end{array}$ & $\begin{array}{c}1.207 \\
677.57 \\
8.3 \%\end{array}$ \\
\hline $500 \mathrm{k}$ & $\begin{array}{c}3.050 \\
2324.32 \\
15.1 \%\end{array}$ & $\begin{array}{c}2.830 \\
3083.26 \\
14.4 \%\end{array}$ & $\begin{array}{c}2.829 \\
3083.98 \\
14.3 \%\end{array}$ & $\begin{array}{c}2.573 \\
1356.57 \\
7.4 \%\end{array}$ \\
\hline $1 \mathrm{M}$ & $\begin{array}{c}6.383 \\
5557.35 \\
16.4 \%\end{array}$ & $\begin{array}{c}5.885 \\
6622.30 \\
15.0 \%\end{array}$ & $\begin{array}{c}5.857 \\
6580.54 \\
14.8 \%\end{array}$ & $\begin{array}{c}5.268 \\
2821.88 \\
7.3 \%\end{array}$ \\
\hline $\begin{array}{l}\mathrm{LLC}= \\
\mathrm{SSMP}\end{array}$ & level ca & the & 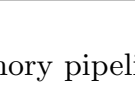 & \\
\hline
\end{tabular}

Table 3: Benchmark 1 on Intel i7: Average CPU time to compute a single time step, LLC misses and SSMP percentage in a four-thread system for several mesh granularities of a single-layered domain using polynomial order 5 . 


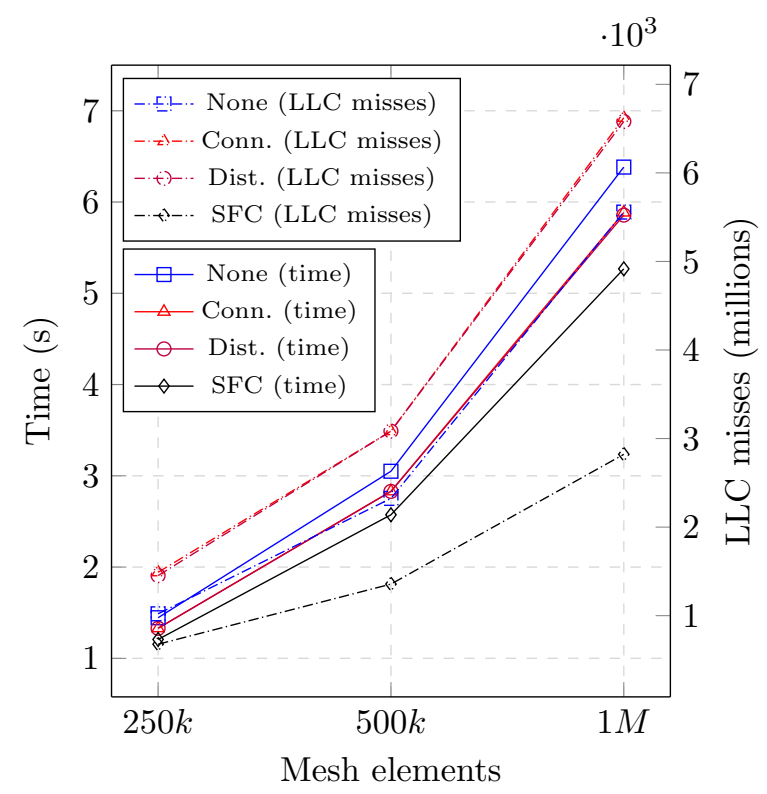

Figure 5: Benchmark 1 on Intel i7: Average CPU time to compute a single time step and LLC misses in a four-thread system for several mesh granularities of a single-layered domain using polynomial order 5 . Values are shown in Table 3.

Mesh elements are in logarithmic scale.

\subsubsection{Multilayered Domain}

In the second benchmark, divided into three parts following those of the first benchmark, we simulated the propagation of an acoustic wave in a multilayered domain with six different layers of varying geometric complexity and properties. Defining the size of an element as the length of its largest edge, the variation in element sizes in the meshes used for the second benchmark was much higher than that of the meshes used in the first benchmark, as shown in Table 4 and Figure 6. As before, the domain was 2000 units wide and 1000 units deep. In the second benchmark, the first mesh had 12 colors and depth 16, and the third and second meshes had 11 colors and depth 18. The purpose of this benchmark was to assess whether larger differences in element sizes would lead to significant leaps between spatially distant elements when traversing them through SFC-based orderings, such that these leaps would translate into worse data locality and execution performance.

\begin{tabular}{ccc}
\hline & \multicolumn{2}{c}{$\begin{array}{c}\text { Standard deviation } \\
\text { in element size, }\end{array}$} \\
& $\begin{array}{c}\text { ratio between maximum } \\
\text { and minimum element size }\end{array}$ \\
\cline { 2 - 3 } Mesh & Benchmark & Benchmark \\
Elements & 1 & 2 \\
\hline \multirow{2}{*}{$250 \mathrm{k}$} & 0.58, & 3.79, \\
& 2.63 & 62.01 \\
$500 \mathrm{k}$ & 0.41, & 2.51, \\
& 2.61 & 55.99 \\
$1 \mathrm{M}$ & 0.29, & 1.55, \\
& 2.81 & 29.91 \\
\hline
\end{tabular}

Table 4: Variation in element sizes in the first and second benchmarks for several mesh granularities.

The CPU times for the first set of benchmarks, testing different mesh granularities and reordering strategies for different thread counts using polynomial order 5 , are listed in Table 5. They should be compared to the corresponding Table 1 of the first benchmark. Figure 7 shows the times and speedups of Table 5, whereas Figure 8 compares the times per element and speedups between the two benchmarks. SFC-based orderings maintained the clear performance advantage seen in the first benchmark, but to a smaller extent. This is caused by the greater variation in element sizes in the meshes of the second benchmark: upon following the steps of the Hilbert curve to iterate between two elements, if these elements are spatially distant from each other, the number of elements in the vicinity of the second element sharing nodes with the first element is likely to be lower. Therefore, significant chunks of the node data loaded in cache lines for the previous element cannot be used by the elements following it, causing cache misses and worse memory efficiency as new nodes need to be read into cache to process the next element.

The CPU timings for different polynomial orders and mesh granularities for the second part of the second benchmark can be seen in Table 6 and Figure 9. Once again, the SFC-based ordering was the fastest 


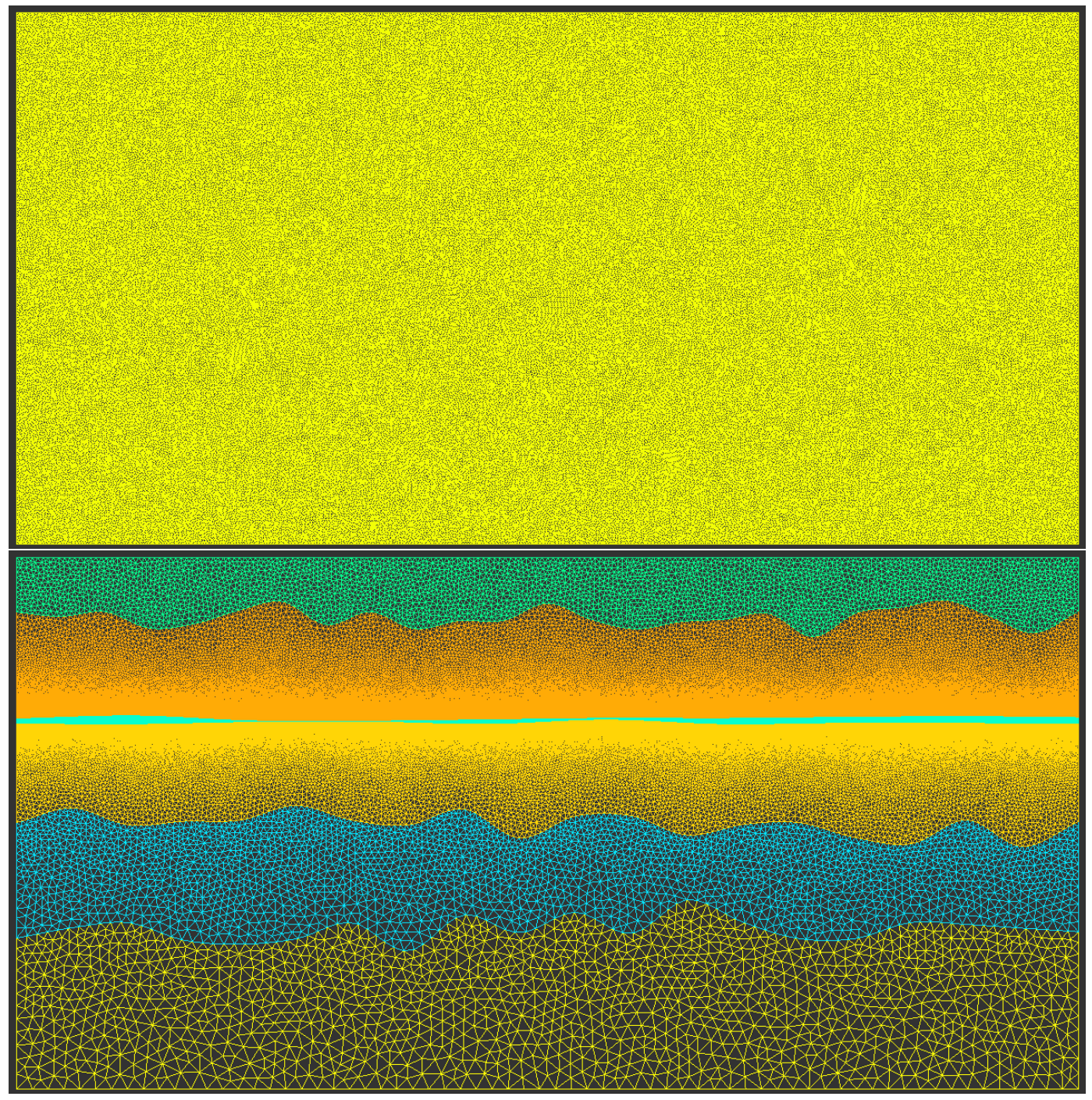

Figure 6: Meshes with 250 thousand elements used in Benchmark 1 (above) and Benchmark 2 (below). Whereas element sizes in the meshes used in the first benchmark were largely homogeneous, in the second benchmark element sizes had much higher variation.

strategy, running between $9.3 \%$ (500 thousand elements, order 5 ) and $20.6 \%$ (250 thousand elements, order 7) faster than the "None" strategy. When comparing Tables 6 and 2, the overall observation is a moderate increase in nearly all compute times. This further emphasizes that the greater variation in ele- ment sizes was harmful for all strategies in relation to the first benchmark. Tables 6 and 2 also show that the computation work per element is considerably higher for higher polynomial orders. Our tests gave no indication that this higher computational work per element would reduce the dependence of the computational 


\begin{tabular}{|c|c|c|c|c|c|c|c|c|c|c|c|c|}
\hline \multirow[b]{4}{*}{ Threads } & \multicolumn{12}{|c|}{ Average CPU time (s) per time step } \\
\hline & \multicolumn{4}{|c|}{ 250k-element domain } & \multicolumn{4}{|c|}{ 500k-element domain } & \multicolumn{4}{|c|}{ 1M-element domain } \\
\hline & \multicolumn{4}{|c|}{ Reordering strategy } & \multicolumn{4}{|c|}{ Reordering strategy } & \multicolumn{4}{|c|}{ Reordering strategy } \\
\hline & None & Conn. & Dist. & $\mathrm{SFC}$ & None & Conn. & Dist. & SFC & None & Conn. & Dist. & $\mathrm{SFC}$ \\
\hline 1 & 5.307 & 5.721 & 5.696 & 4.998 & 11.025 & 11.769 & 11.719 & 10.282 & 22.205 & 23.433 & 23.334 & 20.361 \\
\hline 2 & 4.203 & 4.439 & 4.417 & 4.070 & 8.608 & 8.992 & 8.992 & 8.311 & 17.369 & 18.029 & 17.995 & 16.517 \\
\hline 4 & 2.331 & 2.390 & 2.373 & 2.170 & 4.867 & 4.839 & 4.865 & 4.449 & 9.802 & 9.622 & 9.654 & 8.770 \\
\hline 8 & 1.341 & 1.283 & 1.268 & 1.169 & 2.723 & 2.647 & 2.651 & 2.451 & 5.505 & 5.159 & 5.166 & 4.814 \\
\hline 16 & 0.745 & 0.694 & 0.675 & 0.620 & 1.502 & 1.463 & 1.439 & 1.320 & 3.030 & 2.779 & 2.795 & 2.544 \\
\hline 32 & 0.429 & 0.403 & 0.383 & 0.364 & 0.882 & 0.876 & 0.836 & 0.800 & 1.774 & 1.646 & 1.667 & 1.481 \\
\hline
\end{tabular}

Table 5: Benchmark 2 on Intel Xeon: Average CPU time to compute a single time step in a 32-thread system for several thread counts and mesh granularities of a multilayered domain using polynomial order 5 .
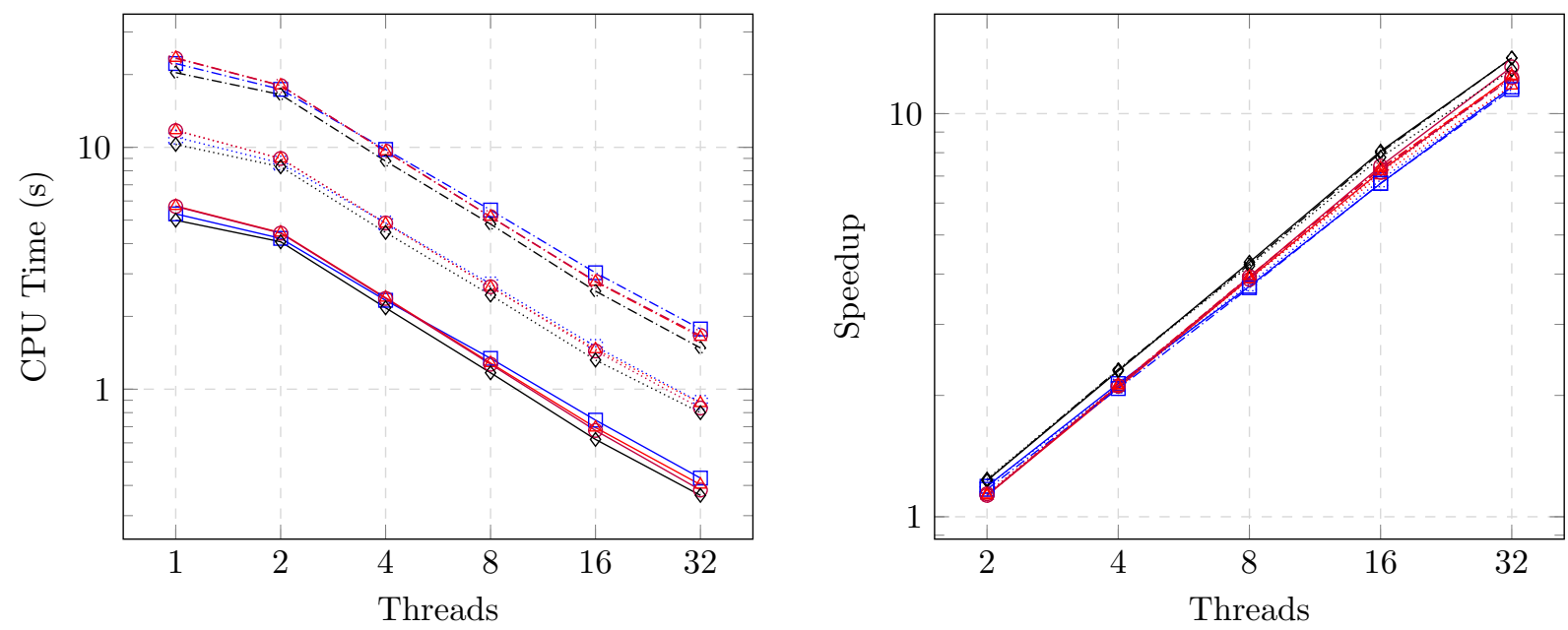

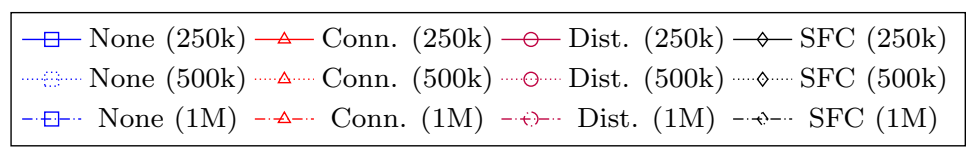

Figure 7: Benchmark 2 on Intel Xeon: Average CPU time to compute a single time step (left) and average speedup in relation to the fastest serial time (right) for several thread counts and mesh granularities of a multilayered domain using polynomial order 5. Values are shown in Table 5. Time, speedup and threads are in logarithmic scale.

performance on the element ordering. We can most likely attribute this to the fact that, for higher-order elements, the data volume for gather and scatter operations is also higher.

The final part of the second benchmark, which measured average CPU times, LLC misses and SSMP percentages in an Intel i7 four-thread system, produced the results seen in Table 7 and Figure 10. The "SFC" strategy once again performed the best in all metrics. When comparing Tables 7 and 3, we find the execution times of Benchmark 2 to be slightly slower. 


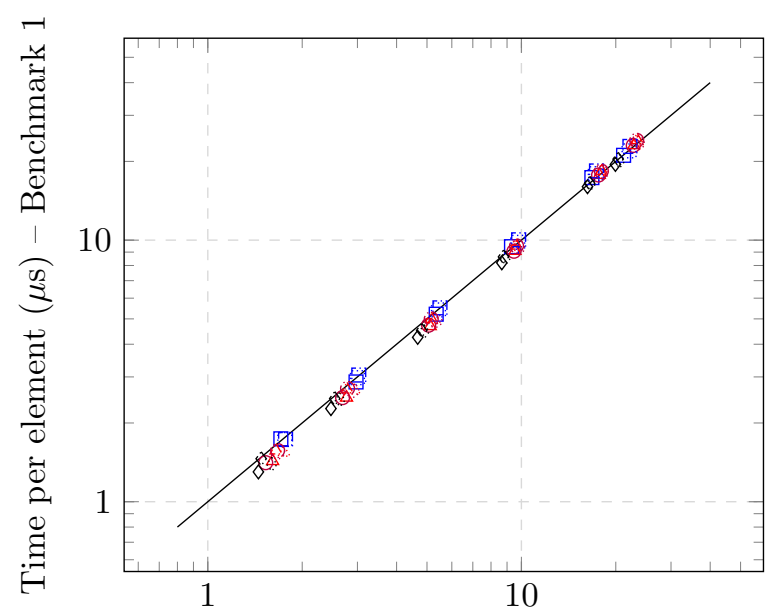

Time per element $(\mu \mathrm{s})$ - Benchmark 2

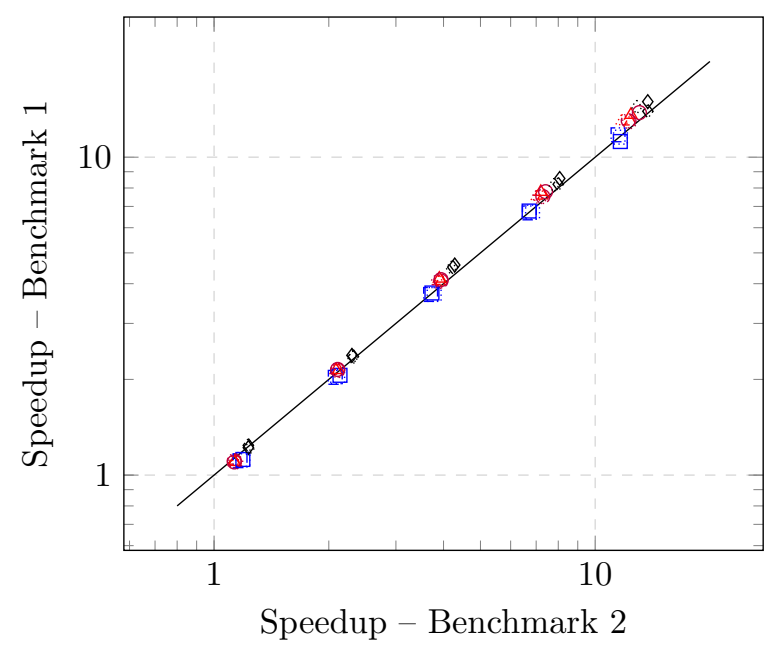

Speedup - Benchmark 2

$$
\begin{aligned}
& \square \text { None }(250 \mathrm{k}) \triangle \text { Conn. }(250 \mathrm{k}) \circ \text { Dist. }(250 \mathrm{k}) \diamond \mathrm{SFC}(250 \mathrm{k}) \\
& \text { None }(500 \mathrm{k}) \diamond \text { Conn. }(500 \mathrm{k}) \text { Dist. }(500 \mathrm{k}) \diamond \mathrm{SFC}(500 \mathrm{k}) \\
& \square \text { None }(1 \mathrm{M}) \triangleleft \text { Conn. }(1 \mathrm{M}) \diamond \text { Dist. }(1 \mathrm{M}) \diamond \mathrm{SFC}(1 \mathrm{M})
\end{aligned}
$$

Figure 8: Execution time per element for Benchmark 1 versus Benchmark 2 (left) and average speedup in relation to the best serial time for Benchmark 1 versus Benchmark 2 (right), on Intel Xeon, for several thread counts and mesh granularities using polynomial order 5. Overall, the times per element are higher in Benchmark 2 and the speedups of SFC-based ordering are higher in Benchmark 1. The diagonal lines show

\begin{tabular}{|c|c|c|c|c|c|c|c|c|c|c|c|c|}
\hline \multirow[b]{4}{*}{ Order } & \multicolumn{12}{|c|}{$\begin{array}{l}\text { Average CPU time (s) per time step, } \\
\text { percentage of time in relation to "None" reordering strategy }\end{array}$} \\
\hline & \multicolumn{4}{|c|}{ 250k-element domain } & \multicolumn{4}{|c|}{ 500k-element domain } & \multicolumn{4}{|c|}{ 1M-element domain } \\
\hline & \multicolumn{4}{|c|}{ Reordering strategy } & \multicolumn{4}{|c|}{ Reordering strategy } & \multicolumn{4}{|c|}{ Reordering strategy } \\
\hline & None & Conn. & Dist. & $\mathrm{SFC}$ & None & Conn. & Dist. & $\mathrm{SFC}$ & None & Conn. & Dist. & $\mathrm{SFC}$ \\
\hline 5 & $\begin{array}{c}0.429 \\
-\end{array}$ & $\begin{array}{l}0.403 \\
93.8 \%\end{array}$ & $\begin{array}{l}0.383 \\
89.1 \%\end{array}$ & $\begin{array}{l}0.364 \\
84.7 \%\end{array}$ & $\begin{array}{c}0.882 \\
-\end{array}$ & $\begin{array}{l}0.876 \\
99.3 \%\end{array}$ & $\begin{array}{l}0.836 \\
94.7 \%\end{array}$ & $\begin{array}{l}0.800 \\
90.7 \%\end{array}$ & 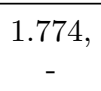 & $\begin{array}{l}1.646, \\
92.8 \%\end{array}$ & $\begin{array}{l}1.667 \\
94.0 \%\end{array}$ & $\begin{array}{l}1.481 \\
83.5 \%\end{array}$ \\
\hline 6 & 0.681 & $\begin{array}{l}0.600 \\
88.2 \%\end{array}$ & $\begin{array}{l}0.594 \\
87.2 \%\end{array}$ & $\begin{array}{l}0.556 \\
81.7 \%\end{array}$ & $\begin{array}{c}1.388 \\
-\end{array}$ & $\begin{array}{l}1.368 \\
98.5 \%\end{array}$ & $\begin{array}{l}1.325 \\
95.4 \%\end{array}$ & $\begin{array}{l}1.216, \\
87.6 \%\end{array}$ & $\begin{array}{c}2.804, \\
-\end{array}$ & $\begin{array}{l}2.607 \\
92.9 \%\end{array}$ & $\begin{array}{l}2.559 \\
91.3 \%\end{array}$ & $\begin{array}{l}2.364, \\
84.3 \%\end{array}$ \\
\hline 7 & $\begin{array}{c}1.027 \\
-\end{array}$ & $\begin{array}{l}0.886 \\
86.3 \%\end{array}$ & $\begin{array}{l}0.883 \\
86.0 \%\end{array}$ & $\begin{array}{l}0.815 \\
79.4 \%\end{array}$ & $\begin{array}{c}2.103, \\
-\end{array}$ & $\begin{array}{l}2.085 \\
99.1 \%\end{array}$ & $\begin{array}{l}1.979 \\
94.1 \%\end{array}$ & $\begin{array}{l}1.825 \\
86.8 \%\end{array}$ & $\begin{array}{c}4.223, \\
-\end{array}$ & $\begin{array}{l}3.921 \\
92.9 \%\end{array}$ & $\begin{array}{l}3.873 \\
91.7 \%\end{array}$ & $\begin{array}{l}3.579, \\
84.8 \%\end{array}$ \\
\hline
\end{tabular}
where the measurements of Benchmarks 1 and 2 would be located if both benchmarks had identical performance. Time per element and speedup are in logarithmic scale.

Table 6: Benchmark 2 on Intel Xeon: Average CPU time to compute a single time step and percentage of time in relation to "None" reordering strategy, for several polynomial orders and mesh granularities of a multilayered domain using 32 threads. 


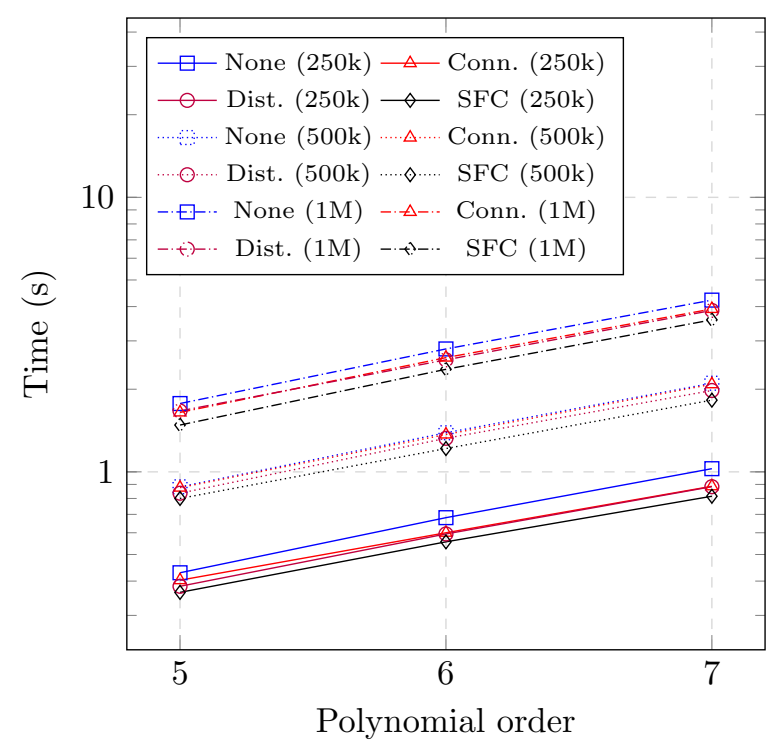

Figure 9: Benchmark 2 on Intel Xeon: Average CPU time to compute a single time step for several polynomial orders and mesh granularities of a multilayered domain using 32 threads. Values are shown in Table 6. Time is in logarithmic scale.

\subsection{Realistic Case Simulation}

Finally we demonstrate the application of the discussed SEM to 2D problems such as synthetic seismic data analysis (Yilmaz, 2001) or microseismic data migration (Trojanowski and Eisner, 2017). We simulated the propagation of a point source with Ricker wavelet profile (Ricker, 1944) in a multilayered domain, 2000 units wide and 1000 units deep (see Figure 11), using a one-million element mesh. Each of the six layers of this domain had a different medium density $\rho$ and compression modulus $K$, and therefore a different wave velocity $v_{p}=\sqrt{K / \rho}$. The wave velocities at the deeper layers were higher than those at the shallower layers (see Table 8). Element size in each layer was chosen proportional to the wave speed of this layer, resulting in a (mostly) constant time step size across the mesh to meet the CFL condition (Courant et al., 1967) for stability of the Heun scheme. The simulation required 197 hours of computing time of 32 threads (sixteen physical cores and

\begin{tabular}{|c|c|c|c|c|}
\hline \multirow[b]{3}{*}{$\begin{array}{l}\text { Domain } \\
\text { elements }\end{array}$} & \multirow{2}{*}{\multicolumn{4}{|c|}{$\begin{array}{l}\text { Average CPU time (s), } \\
\text { LLC misses (millions), } \\
\text { SSMP percentage } \\
\text { Reordering strategy }\end{array}$}} \\
\hline & & & & \\
\hline & None & Conn. & Dist. & SFC \\
\hline $250 \mathrm{k}$ & $\begin{array}{c}1.483 \\
1082.96 \\
15.2 \%\end{array}$ & $\begin{array}{c}1.437 \\
1655.40 \\
15.8 \%\end{array}$ & $\begin{array}{c}1.440 \\
1653.96 \\
14.9 \%\end{array}$ & $\begin{array}{c}1.321 \\
691.25 \\
8.2 \%\end{array}$ \\
\hline $500 \mathrm{k}$ & $\begin{array}{c}3.178 \\
2500.74 \\
15.1 \%\end{array}$ & $\begin{array}{c}3.044 \\
3470.64 \\
15.5 \%\end{array}$ & $\begin{array}{c}3.051 \\
3504.49 \\
14.5 \%\end{array}$ & $\begin{array}{c}2.762, \\
1360.90, \\
7.5 \%\end{array}$ \\
\hline $1 \mathrm{M}$ & $\begin{array}{c}6.340 \\
5632.23 \\
15.3 \%\end{array}$ & $\begin{array}{c}6.041 \\
7145.78 \\
15.1 \%\end{array}$ & $\begin{array}{c}6.051 \\
7129.94 \\
15.5 \%\end{array}$ & $\begin{array}{c}5.503, \\
2808.20, \\
7.4 \%\end{array}$ \\
\hline $\begin{array}{l}\mathrm{LLC}= \\
\mathrm{SSMP}\end{array}$ & vel ce & & & \\
\hline
\end{tabular}

Table 7: Benchmark 2 on Intel i7: Average CPU time to compute a single time step, LLC misses and SSMP percentage in a four-thread system for several mesh granularities of a multilayered domain using polynomial order 5 .

two hardware threads per core), with a time step of 1.2 microseconds.

\begin{tabular}{cc}
\hline Layer & $\begin{array}{c}\text { Wave velocity } \\
\text { (units/s) }\end{array}$ \\
\hline 1 & 1500.0 \\
2 & 2500.0 \\
3 & 2000.0 \\
4 & 2500.0 \\
5 & 3000.0 \\
6 & 4000.0 \\
\hline
\end{tabular}

Table 8: Wave velocities at each layer of the multilayered domain. Geometry shown in Figure 11. Labeling of layers starts from the top.

We placed a single Ricker wavelet source in the center of the domain. Figure 12 illustrates that the shape and motion of the resulting waves behaved as expected, with a noticeably faster propagation speed 


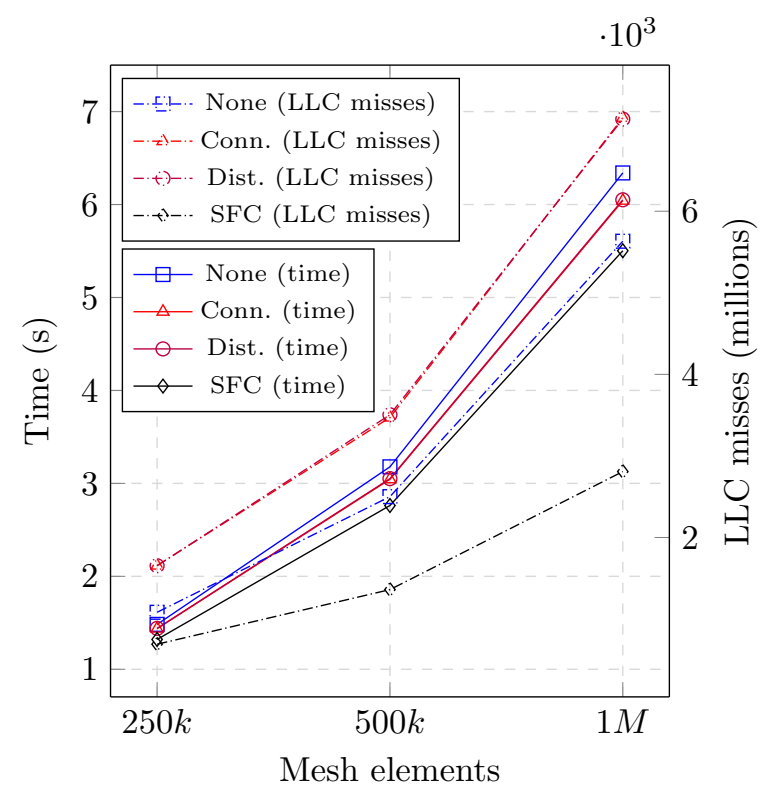

Figure 10: Benchmark 2 on Intel i7: Average CPU time to compute a single time step and LLC misses in a four-thread system for several mesh granularities of a multilayered domain using polynomial order 5 . Values are shown in Table 7 . Mesh elements are in logarithmic scale.

at the deeper layers because of their higher wave velocities. Although absent at the upper boundary of the domain, we observed wave reflections in the lower boundary; they could be dealt with by employing absorbing boundary conditions (Engquist and Majda, 1977). However, as the use of such conditions would require the $\mathrm{PDE}$ coefficients to be complex numbers, we do not address it here.

Although we performed all the experiments presented here with sufficiently small time steps to ensure consistent results, we made no rigorous investigations to establish the conditions for numerical stability.

\section{Conclusions}

In this work, we proposed a memory reordering algorithm based on generalized Hilbert curves for use with the SEM to obtain improved memory efficiency and faster execution speed. We used this algorithm to implement a generic $2 \mathrm{D}$ wave equation solver using the SEM with unstructured meshes and an explicit time integration scheme.

The proposed algorithm derived from Hilbert curves presented the shortest compute time compared to three other commonly used approaches. This was observed not only with several mesh granularities, but also with large variations in element sizes. Simulation time reduced about $20 \%$ when the element size variation was moderate (standard deviation varying between 0.29 and 0.58 ), and about $15 \%$ when size variation was more significant (standard deviation varying between 1.55 and 3.79). This makes the proposed algorithm suitable to situations where specific regions of a mesh may demand particular refinements - for instance, in stratified domains containing thin layers whose strong curvature requires relatively small elements.

In our experiments, memory reordering increased mesh preparation time by a value between $15 \%$ and $20 \%$. In the complete simulation presented in Section 6.2, mesh preparation time was negligible within the total execution time, which is the case for most application scenarios with large unstructured meshes. Therefore, applying SFC-based memory reordering in all practical applications has a potential run time reduction of $25 \%$, as we have demonstrated, and hardly any downside.

We point out some suggestions for future work. Firstly, there is still room to improve the performance of the arithmetic code in Eqs. (15) and (12), in particular by precalculating more values. For instance, we can compute the multiplications between basis function derivatives and arrays $F K / B K$ before time integration starts, at the cost of an increase in memory consumption and additional memory to cache data traffic for the precalculated values. Secondly, the SEM formulation shown here can be readily extended to $3 \mathrm{D}$ by defining the continuous variable $u$ as a vector and modifying Eqs. (4) and (5) accordingly. Luo and Pozrikidis (2006) provide local node locations and basis functions derived from Lobatto polynomials over tetrahedra that are well-suited for the SEM in the 3D case. 


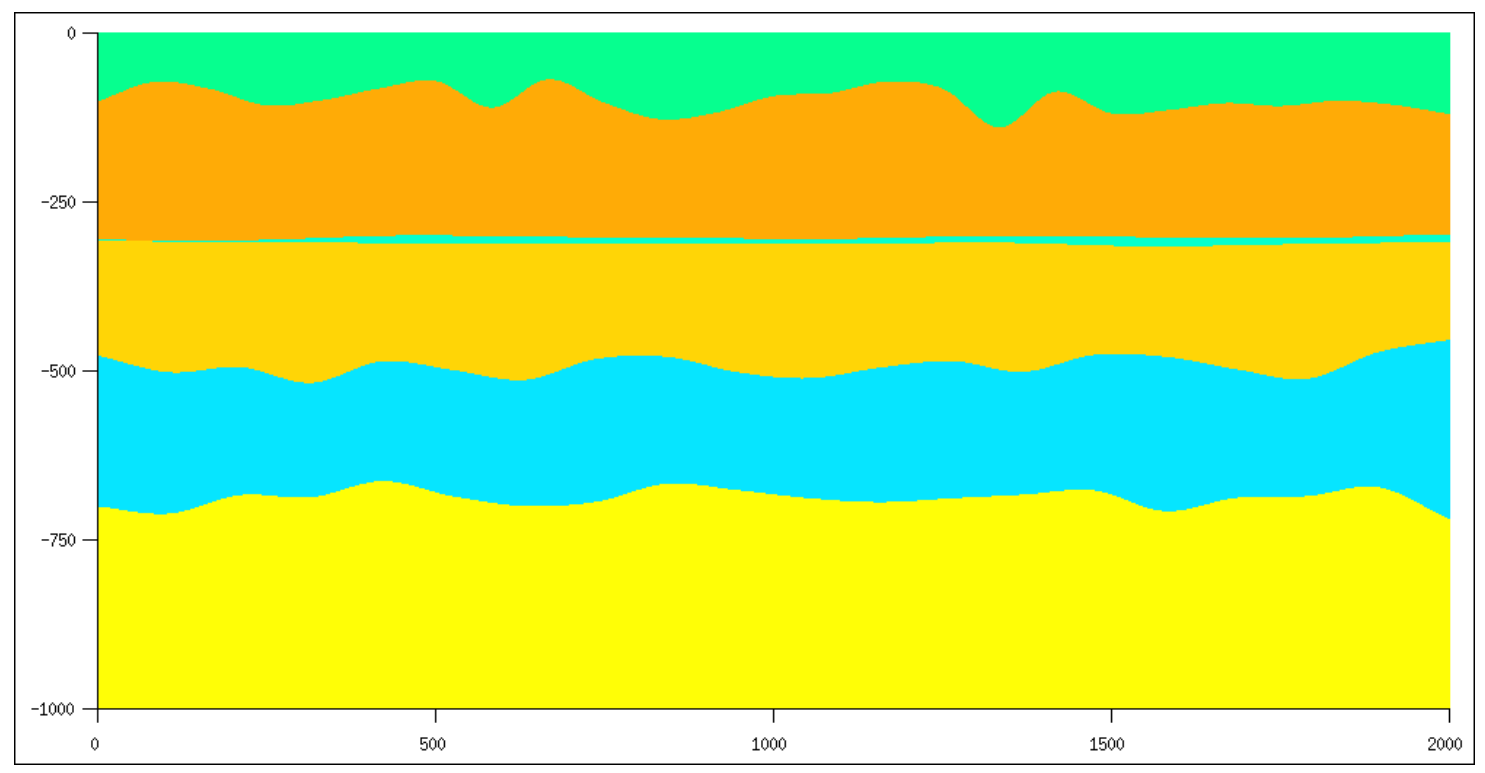

Figure 11: A multilayered domain. The layer labeling starts from the top with several wave velocities shown in Table 8. The wave velocities at the deeper layers are higher than those at the shallower layers.

\section{Declarations}

This research did not receive any specific grant from funding agencies in the public, commercial, or notfor-profit sectors.

\section{Acknowledgments}

We would like to thank Adam Ellery of the University of Queensland for his instrumental help and advice regarding SEM and basis functions.

This research was supported by the High Performance Computing Center at UFRN (NPAD/UFRN).

\section{Computer Code Availability}

The computer code produced in this research (version v20210104) is available for download at https:// gitlab.com/lappsufrn/shirley under Apache License 2.0 .

\section{References}

Afanasiev, M. et al. (2018). "Modular and flexible spectral-element waveform modelling in two and three dimensions". In: Geophysical Journal International 216.3, pp. 1675-1692. ISSN: 0956-540X. DOI: $10.1093 / \mathrm{gji} / \mathrm{ggy} 469$.

Arvo, J. (1991). Graphics Gems II. Boston, MA: Academic Press, 672pp. Is B N: 978-0080507545.

Bakir, A. C. and R. L. Nowack (2012). "Modeling Seismic Attributes of Pn Waves using the Spectral-Element Method". In: Pure and Applied Geophysics 169.9, pp. 1539-1556. IS N N: 1420-9136. DOI: $10.1007 / \mathrm{s} 00024-011-0414-z$.

Blyth, M. G. and C. Pozrikidis (2006). "A Lobatto interpolation grid over the triangle". In: IMA Journal of Applied Mathematics 71.1, pp. 153-169. IS S N: 0272-4960. DOI: 10.1093/imamat/hxh077.

Burstedde, C., L. C. Wilcox, and O. Ghattas (2011). "p4est: Scalable Algorithms for Parallel Adaptive Mesh Refinement on Forests of Octrees". In: SIAM Journal on Scientific Computing 33.3, pp. 11031133. DOI: $10.1137 / 100791634$. 

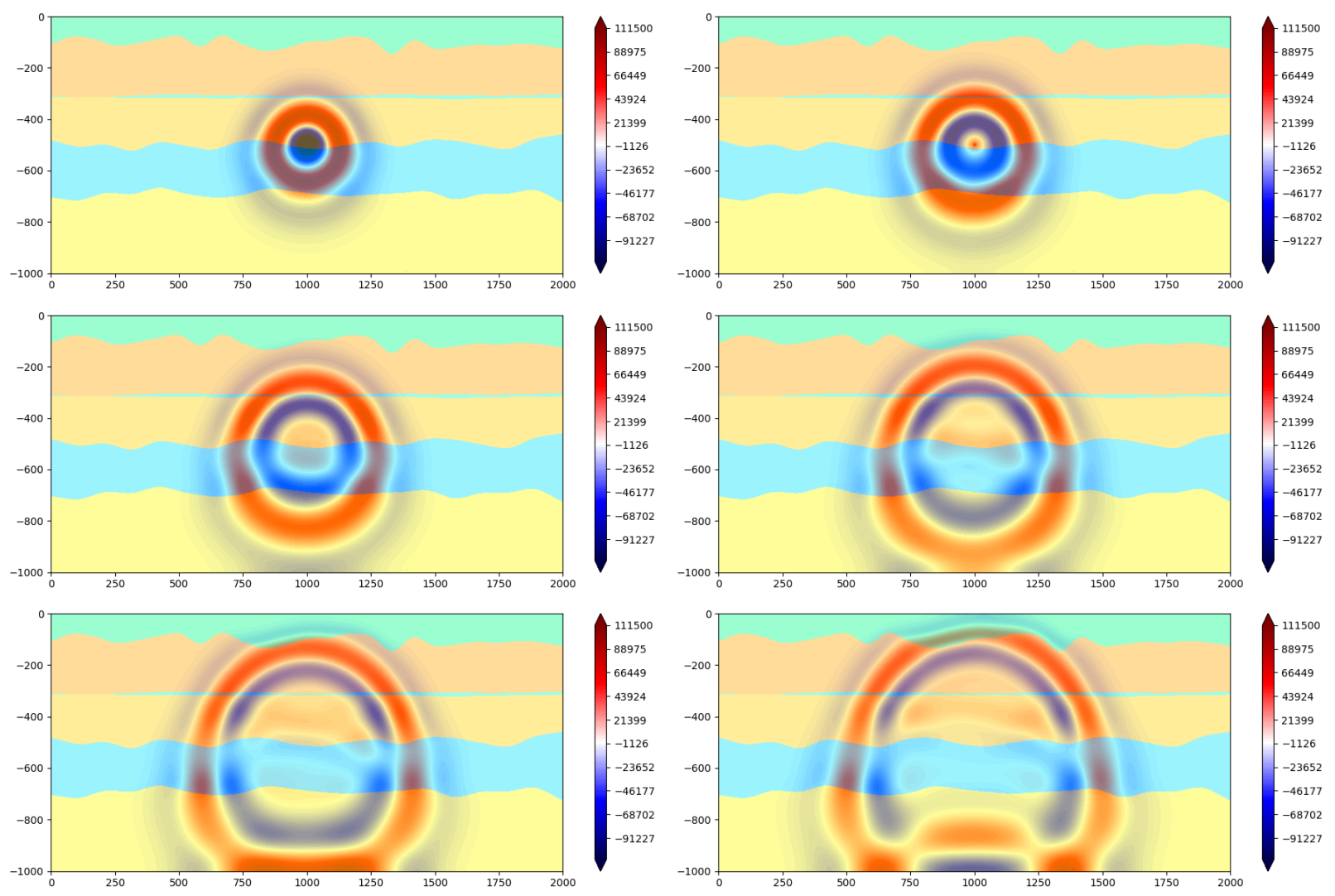

Figure 12: Ricker wavelet propagation in a multilayered domain at $0.275,0.300,0.325,0.350,0.375$ and $0.400 \mathrm{~s}$. The propagation of the wave is noticeably faster at the deeper layers, with the last two figures showing a reflection at the lower boundary of the domain.

Butcher, J. C. (2008). Numerical methods for ordinary differential equations. 2nd ed. Chichester, England; Hoboken, NJ: Wiley, 482pp. Is B N: 978 0470723357.

Chaskalovic, J. (2008). Finite Element Methods for Engineering Sciences: Theoretical Approach and Problem Solving Techniques. 1st ed. Berlin: Springer-Verlag, 267pp. IS B N : 978-3-540-76342-0. D O I: $10.1007 / 978-3-540-76343-7$.

Courant, R., K. Friedrichs, and H. Lewy (1967). "On the Partial Difference Equations of Mathematical Physics". In: IBM Journal of Research and Development 11.2, pp. 215-234. D O I: 10.1147/rd.112. 0215.
Cuthill, E. and J. McKee (1969). "Reducing the Bandwidth of Sparse Symmetric Matrices". In: Proceedings of the 1969 24th National Conference. ACM '69. New York, NY, USA: Association for Computing Machinery, pp. 157-172. ISBN: 9781450374934. D OI: 10.1145/800195.805928.

Davies, M., L. Gross, and H. Muhlhaus (2004). "Scripting high performance Earth systems simulations on the SGI Altix 3700". In: Proceedings. Seventh International Conference on High Performance Computing and Grid in Asia Pacific Region, 2004, pp. 244-251. D O I: 10.1109/HPCASIA . 2004. 1324041. 
Engquist, B. and A. Majda (1977). "Absorbing boundary conditions for numerical simulation of waves". In: Proceedings of the National Academy of Sciences 74.5, pp. 1765-1766. IS S N: 0027-8424. D O I: $10.1073 /$ pnas.74.5.1765.

Feynman, R. P., R. B. Leighton, and M. Sands (2011). The Feynman Lectures on Physics. The New Millenium ed. Vol. I. New York, NY: Basic Books, 560pp. ISBN: 978-0465024933.

Geuzaine, C. and J.-F. Remacle (2009). "Gmsh: A 3-D finite element mesh generator with built-in pre- and post-processing facilities". In: International Journal for Numerical Methods in Engineering 79.11, pp. 1309-1331. DOI: 10 . $1002 / \mathrm{nme}$ 2579.

Heun, K. (1900). "Neue Methoden zur approximativen Integration der Differentialgleichungen einer unabhängigen Veränderlichen". In: Zeitschrift für Mathematik und Physik 45, 23-38 [in German].

Hilbert, D. (1891). "Über die stetige Abbildung einer Linie auf ein Flächenstück". In: Mathematische Annalen 38.3, 459-460 [in German]. Is S N: 1432 1807. D O I: $10.1007 / \mathrm{BF} 01199431$.

Intel (2021). Intel VTune Profiler. Available from: https://software.intel.com/content/www/us/ en/develop/tools / oneapi / components / vtuneprofiler.html, accessed in April 2021.

Komatitsch, D., C. Barnes, and J. Tromp (2000). "Wave propagation near a fluid-solid interface: A spectral-element approach". In: Geophysics 65.2, pp. 623-631. DOI: 10.1190/1.1444758.

Komatitsch, D. and J. Tromp (1999). "Introduction to the spectral element method for threedimensional seismic wave propagation". In: Geophysical Journal International 139.3, pp. 806-822. IS SN: 0956-540X. DOI: $10.1046 / \mathrm{j} .1365-246 \mathrm{x}$. 1999.00967.x.

Komatitsch, D. and J.-P. Vilotte (1998). "The Spectral Element method: an efficient tool to simulate the seismic response of 2D and 3D geological structures". In: Bulletin of Seismological Society of America 88, pp. 368-392.

Komatitsch, D. et al. (2012). SPECFEM3D Cartesian v2.0.2 [software]. Available from: https: //geodynamics.org/cig/software/specfem3d/, accessed in April 2021. Computational Infrastructure for Geodynamics.

Kopriva, D. A. (2009). Implementing Spectral Methods for Partial Differential Equations: Algorithms for Scientists and Engineers. 1st ed. Dordrecht: Springer Netherlands, 415pp. Is BN: 9789048122608.

Kutta, M. W. (1901). "Beitrag zur Näherungsweisen Integration Totaler Differentialgleichungen". In: Zeitschrift für Mathematik und Physik 46, 435-453 [in German].

Luo, H. and C. Pozrikidis (2006). "A Lobatto interpolation grid in the tetrahedron". In: IMA Journal of Applied Mathematics 71.2, pp. 298-313. ISSN: 0272-4960. D OI: 10.1093/imamat/hxh111.

Mellor-Crummey, J., D. Whalley, and K. Kennedy (2001). "Improving Memory Hierarchy Performance for Irregular Applications Using Data and Computation Reorderings". In: International Journal of Parallel Programming 29.3, pp. 217247. ISSN: 1573-7640. DOI: 10 . 1023 / A : 1011119519789.

Mercerat, E. D., J.-P. Vilotte, and F. J. SánchezSesma (2006). "Triangular spectral element simulation of two-dimensional elastic wave propagation using unstructured triangular grids". In: Geophysical Journal International 166.2, pp. 679-698. D O I: 10.1111/j.1365-246X.2006.03006.x.

Morton, G. M. (1966). A computer oriented geodetic data base and a new technique in file sequencing. Available from: https://dominoweb.draco.res. ibm . com/reports/Morton1966.pdf, accessed in April 2021.

OpenMP (2021). The OpenMP API specification for parallel programming. Available from: https : // www . openmp.org, accessed in April 2021.

Patera, A. T. (1984). "A Spectral Element Method for Fluid Dynamics: Laminar Flow in a Channel Expansion". In: Journal of Computational Physics 54.3, pp. 468-488. ISSN: 0021-9991. DOI: 10 . 1016/0021-9991 (84) 90128-1.

Peano, G. (1890). "Sur une courbe, qui remplit toute une aire plane". In: Mathematische Annalen 36.1, 157-160 [in French]. ISSN: 1432-1807. DOI: 10 . $1007 /$ BF01199438. 
Ricker, N. (1944). "Wavelet functions and their polynomials". In: Geophysics 9.3, pp. 314-323. IS S N 0016-8033. DOI: 10.1190/1.1445082.

Runge, C. (1895). "Über die numerische Auflösung von Differentialgleichungen". In: Mathematische Annalen 46.2, 167-178 [in German]. DOI: 10 . $1007 /$ BF01446807.

Sastry, S. P. et al. (2014). "Improved cache utilization and preconditioner efficiency through use of a space-filling curve mesh element- and vertexreordering technique". In: Engineering with Computers 30.4 , pp. 535-547. ISSN: 1435-5663. DOI: $10.1007 / \mathrm{s} 00366-014-0363-0$.

Schaa, R., L. Gross, and J. du Plessis (2016). "PDEbased geophysical modelling using finite elements: examples from 3D resistivity and 2D magnetotellurics". In: Journal of Geophysics and Engineering 13 (2), S59-S73. D O I: 10.1088/1742-2132/13/2/ S59.

Stallings, W. (2009). Computer Organization and Architecture: Designing for Performance. 8th ed. Upper Saddle River, NJ: Prentice Hall, 792pp. ISBN: 978-0136073734.

Trojanowski, J. and L. Eisner (2017). "Comparison of migration-based location and detection methods for microseismic events". In: Geophysical Prospecting 65.1, pp. 47-63. D O I: 10.1111/1365-2478.12366.

Červený, J. (2018). Generalized Hilbert ("gilbert") space-filling curve for rectangular domains of arbitrary (non-power of two) sizes. Available from: https : / / github . com / jakubcerveny / gilbert, accessed in April 2021.

Warren, H. S. (2013). Hacker's Delight. 2nd ed. Upper Saddle River, NJ: Addison-Wesley, 494pp. IS B N : 978-0-321-84268-8.

Yilmaz, Ö. (2001). Seismic data analysis: processing, inversion, and interpretation of seismic data. 2nd ed. Tulsa, OK: Society of Exploration Geophysicists, 2027pp. IS B N : 978-1560800941.

Zampieri, E. and L. F. Pavarino (2006). "Approximation of acoustic waves by explicit Newmark's schemes and spectral element methods". In: Journal of Computational and Applied Mathematics 185.2. Special Issue: International Workshop on the Technological Aspects of Mathematics, pp. 308-325.
ISSN: 0377-0427. DOI: 10.1016/j.cam.2005. 03 . 013. 